\title{
A Review of Some Recent Work on Mid-Latitude Spread-F Occurrence as Detected by Ionosondes
}

\author{
G. G. BOWMAN \\ Department of Physics, The University of Queensland, Brisbane, Q. 4067, Australia
}

(Received April 10, 1989; Revised November 8, 1989)

\begin{abstract}
The characteristics of mid-latitude spread- $F$ occurrence are reviewed (in the light of some recent publications) in terms of four different scale sizes. The term "macroscale" has been used to describe significant height rises and electron-density depletions which in general precede spread- $F$ occurrence. TID wavetrains in the form of wavelike modulations of these height rises are termed "large-scale" structures. The experimental evidence suggests that off-vertical reflections from tilts associated with these wavetrains are responsible for the basic components of spread traces on ionograms. Also, it is proposed that "medium-scale" structures at the $F_{2}$-layer level are a result of stratification effects, and finally that field-aligned irregularities which sometimes produce weak spread traces, be termed "small-scale" structures. Attention is drawn to the fact that structures similar to those proposed here for mid-latitude spread- $F$ appear to be related to other phenomena in equatorial and sub-auroral zone regions. It is suggested that the breaking of atmospheric gravity waves has a central role to play in the development of these mid-latitude spread- $F$ structures.
\end{abstract}

\section{Introduction}

The extra diffuse traces (supplementary to the main trace) which were detected on ionograms in the early days of ionospheric research were classified as spread- $F$ traces (BERKNER and WELLS, 1934; BOOKER and WELLS, 1938). Since researchers at the time were completely unaware of the existence of travelling ionospheric disturbances (TIDs) they ascribed this phenomenon to scattering from small-scale ionospheric structures (plasma instabilities) embedded in an otherwise horizontally stratified ionosphere. However, evidence for the presence of large-scale structures at the times of spread- $F$ occurrence has been available from the 1950 s onwards, thus giving support to the concept that much larger structures are involved (ROY and VERMA, 1953; UYEDA and OGATA, 1954; BOWMAN, 1960a, 1968a, 1981; KLEMPERER, 1963; KING, 1970). In addition MCNICOL et al. (1956), using a set of spaced stations, detected 87 TIDs (speeds 41 to $97 \mathrm{~ms}^{-1}$ ) travelling generally in north-west directions and intimately associated with mid-latitude spread- $F$ occurrence. Despite this experimental evidence, the original ideas involving a scattering mechanism became well-established in the minds of researchers, so that it is only in more recent times that large-scale structures have had reasonable support as the primary cause of the spread- $F$ traces (see e.g., BOOKER, 1979; BOOKER et al., 1986; RASTOGI, 1989). Furthermore, for a low-latitude station KAUSHIKA and DE MENDONCA (1974) have 
reported events representing periodicities of some tens of minutes in the ionospheric total electron content (TEC) (using VHF signals from a geostationary satellite) which were coincident with the occurrence of spread- $F$ on bottomside ionograms (see also LEI et al., 1986).

The associations which were established some time ago between radio-wave scintillations of transmissions from extraterrestrial sources as well as from artificialearth satellites (see e.g., BRIGGS, 1964) and spread- $F$ occurrence seemed to support the scattering mechanism. Field-aligned columns have also been considered in the past (HERMAN, 1966) for the small-scale structures which have been proposed responsible for spread- $F$. At the present time, although as stated large-scale structures appear primarily responsible for spread- $F$, there seems little doubt that small-scale structures are also present at times of spread- $F$ occurrence (especially in the form of field-aligned irregularities - to be discussed later). However, the evidence suggests that the contribution of these small-scale structures to the extra traces on ionograms (defined as spread- $F$ traces) is minimal. Consistent with the foregoing statements is the fact that wavelike patterns in the TEC readings deduced from Faraday rotation measurements are found to accompany short-period scintillations (SINNO and KAN, 1978) and also spread- $F$ occurrence (LEI et al., 1986). SINNO and KAN (1978) report "irregular fluctuations of Faraday rotation angle ... with periods a few minutes up to several tens of minutes .... At the same time, severe intensity scintillation of period usually shorter than one minute occurred" (see also KERSLEY et al., 1980). RASTOGI et al. (1989) find short-period scintillations to be associated with range-type spread- $F$, which they claim is "characterised by a series of discrete multiple layers parallel to the first order $p^{\prime}-f$ trace over the entire frequency range". Thus, scintillation recordings can be used as a measure of spread$F$ occurrence (see e.g., RODGER and AARONS, 1988).

The evidence for large-scale structures comes primarily from mid-latitude regions, so that this paper will concentrate on spread- $F$ recorded in these regions. There is however experimental evidence to support (for spread- $F$ ionograms) the large-scale hypothesis for other latitude regions (BOWMAN, 1968b; ARGO and KELLEY, 1986). If it is accepted that the basic components of a nighttime spread- $F$ ionogram are strong duplicate traces at extra ranges (the evidence is welldocumented and summarized by BowMAN, 1988a) then the most obvious explanation is that a duplicate trace results from an off-vertical reflection from a horizontally-displaced tilted surface. Furthermore, the recording of such duplicate traces requires that radiation at an oblique angle to the vertical traverses an ionospheric electron-density profile not unlike that encountered by the verticallyincident radiation. It would just not seem possible that plasma instabilities (considered in some detail by FEJER and KELLEY, 1980) whether distributed randomly or not, could be responsible for strong traces at excess ranges which are close replicas of the main trace. Extra traces are also found on daytime ionograms resulting from the passage of TIDs (BowMAN et al., 1987). However, these daytime extra traces are not in general replicas of the main trace; they are often seen as distorted traces. The main trace can also suffer distortion. Arguments will presented for considering these daytime traces as also part of the spread- $F$ phenomenon.

This present paper will review some recent work which (together with other experimental evidence) supports the subdivision of (at least nighttime) irregularities 
detected by ionograms at times of spread- $F$ occurrence into four distinct types. These will be classified in terms of structure sizes by the terms "macroscale", "largescale", "medium-scale" and "small-scale". The last three types mentioned will be considered initially and largely in terms of material already published. After this, additional new evidence for macroscale structures at times of mid-latitude nighttime spread- $F$ occurrence will be considered. In addition, comparisons will be made with macroscale structures associated with other ionospheric phenomena.

The data analysed for this paper came from recordings by a modern ionosonde (called a disonde-BowmAN et al., 1986) on Bribie Island $\left(27.05^{\circ} \mathrm{S}, 153.16^{\circ} \mathrm{E}\right.$ geographic), and also from a routinely recording ionosonde at Moggill, some $60 \mathrm{~km}$ magnetically south of the Bribie Island site. Both recording sites are near Brisbane, Australia (corrected-geomagnetic coordinates, $36.4^{\circ} \mathrm{S}, 226.6^{\circ} \mathrm{E}-$ HAKURA, 1965). The modern ionosonde recordings were made at 5.2 minute intervals while the Moggill recordings were made every 15 minutes. Some other sundry recordings are also used. The times quoted will be local times ( $\mathrm{LT}=\mathrm{UT}+10 \mathrm{~h})$ and numbers after any decimal point which follows hours and minutes will represent seconds. The disonde is capable of propagating (as well as detecting) O-rays or X-rays separately. The magnetoionic component recorded for particular events will be indicated on the diagrams presented.

\section{Experimental Results}

\subsection{Large-scale structures}

In the 1950's when evidence of TIDs began to emerge, MUNRO (1953) did some simple calculations on the extra traces expected to be seen on ionograms from a single cycle of a passing TID wavetrain. His results show that for ionization distributions representative of nighttime conditions extra traces, which in fact are close duplicates of the main trace, can be expected. However, during daylight hours when there is additional lower-level ionization, the extra traces were distorted and often appeared as cusps attached to the main trace. In the years which followed the discovery of TIDs it was generally thought that TIDs were mainly daytime phenomena, since the extra traces were often not easily identifiable at night. On the other hand, spread- $F$ was regarded as a different phenomenon which occurred during night hours. It now seems likely that these two apparently different phenomena in fact represent different aspects of the same phenomenon. Consistent with this view is the fact that there is a strong correlation between daytime TIDs (as detected by distorted ionogram traces) and nighttime spread- $F$ (see Fig. 2 of BOWMAN et al. (1987)). Figure 1(a) illustrates the nature of the extra ionogram traces observed for both daytime and nighttime recordings.

Because the duplicate traces which make up a range-spread ionogram are subject to modulation due to signal-strength variations along their length as well as sometimes overlapping one another in range (see Fig. 1 of BOWMAN (1981) and Fig. 3 of BowMAN (1987)), on many occasions the spread traces appear diffuse. Sometimes the effects of medium-scale structures (to be considered in the next subsection) result in further modulation of these duplicate traces. The ionogram shown in Fig. 2(a) shows a small section of a duplicate trace (over a frequency span of approximately $300 \mathrm{kHz}$ ) with an excess virtual range of $40 \mathrm{~km}$. In addition, 
IONOSPHERIC IRREGULARITIES

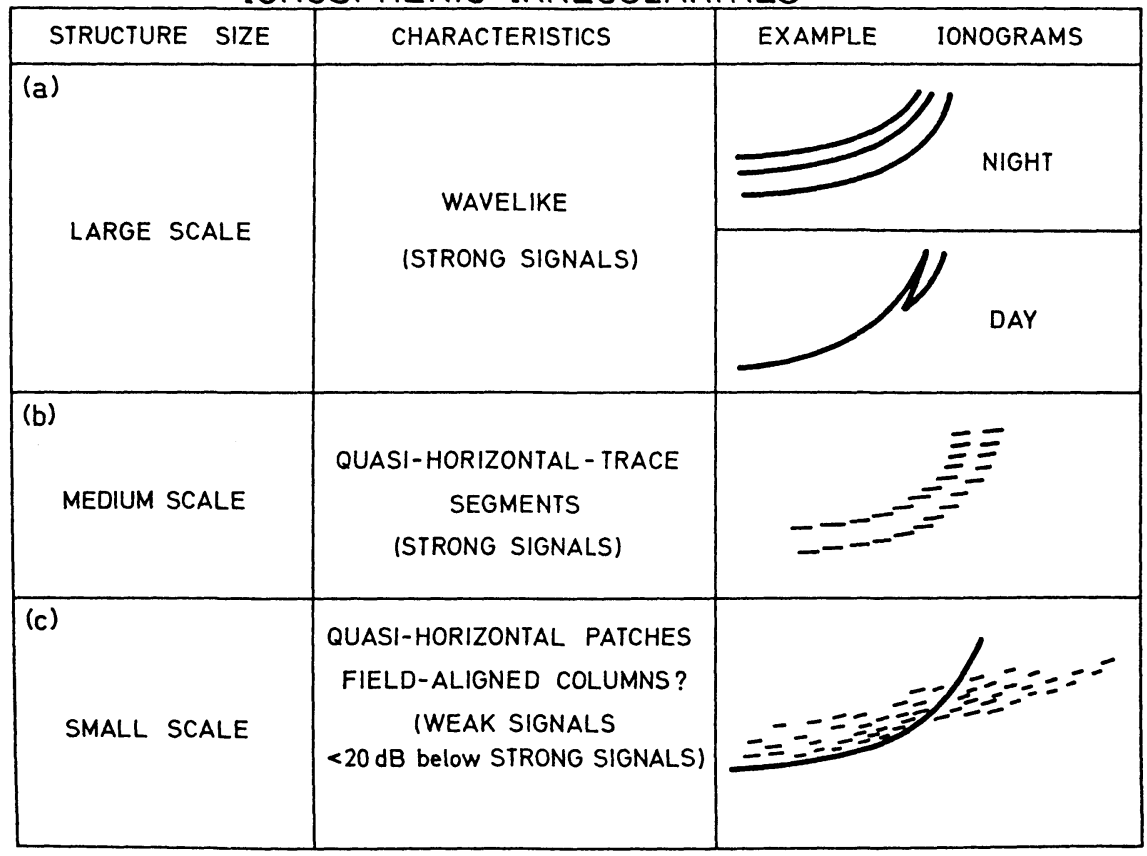

Fig. 1. Diagrammatic illustrations of mid-latitude ionospheric irregularities (a) large scale, (b) medium scale and (c) small scale.

another duplicate trace is so close to the main trace that it overlaps, leading to interference fringes as described by BowMAN (1987). The calculated trace separation of $8.2 \mathrm{~km}$ is consistent with the swept-gain recording on Fig. 2(b) (recorded $12 \mathrm{~s}$ later) where the overlapping trace is clearly identified. Figure 2(c) is a daytime limited-range ionogram showing a V-shaped extra trace which is found to move upwards before disappearing, as predicted and illustrated by LOBB and TITHERIDGE (1977). Similar V-shaped traces are illustrated by Figs. 3(e) to 3(h) of BowMAN et al. (1987) where they are shown to form part of a sequence of ionospheric conditions relating to the passage of large-scale daytime structures associated with TID wavetrains.

\subsection{Medium-scale structures}

Although until recently the use of rockets was the main method of detecting $E$-layer stratifications, it has now been shown (BowMAN, 1989) that ionosondes are capable of recording these even when the vertical separations between the stratifications are as small as $2 \mathrm{~km}$ or less. This $E$-layer stratification is illustrated by Figs. 2(h) and 2(i). Figure 2(g) is the lower-frequency section of a full-range ionogram which includes the $E$-layer section (see arrows) shown in more detail by Figs. 2(h) and 2(i). On Figs. 2(h) and 2(i), which cover a frequency range from 2.3 to $2.9 \mathrm{MHz}$, four stratifications are indicated by the letters, P, Q, R and S. Use has been made of a method of determining with reasonable accuracy virtual heights of traces from the 

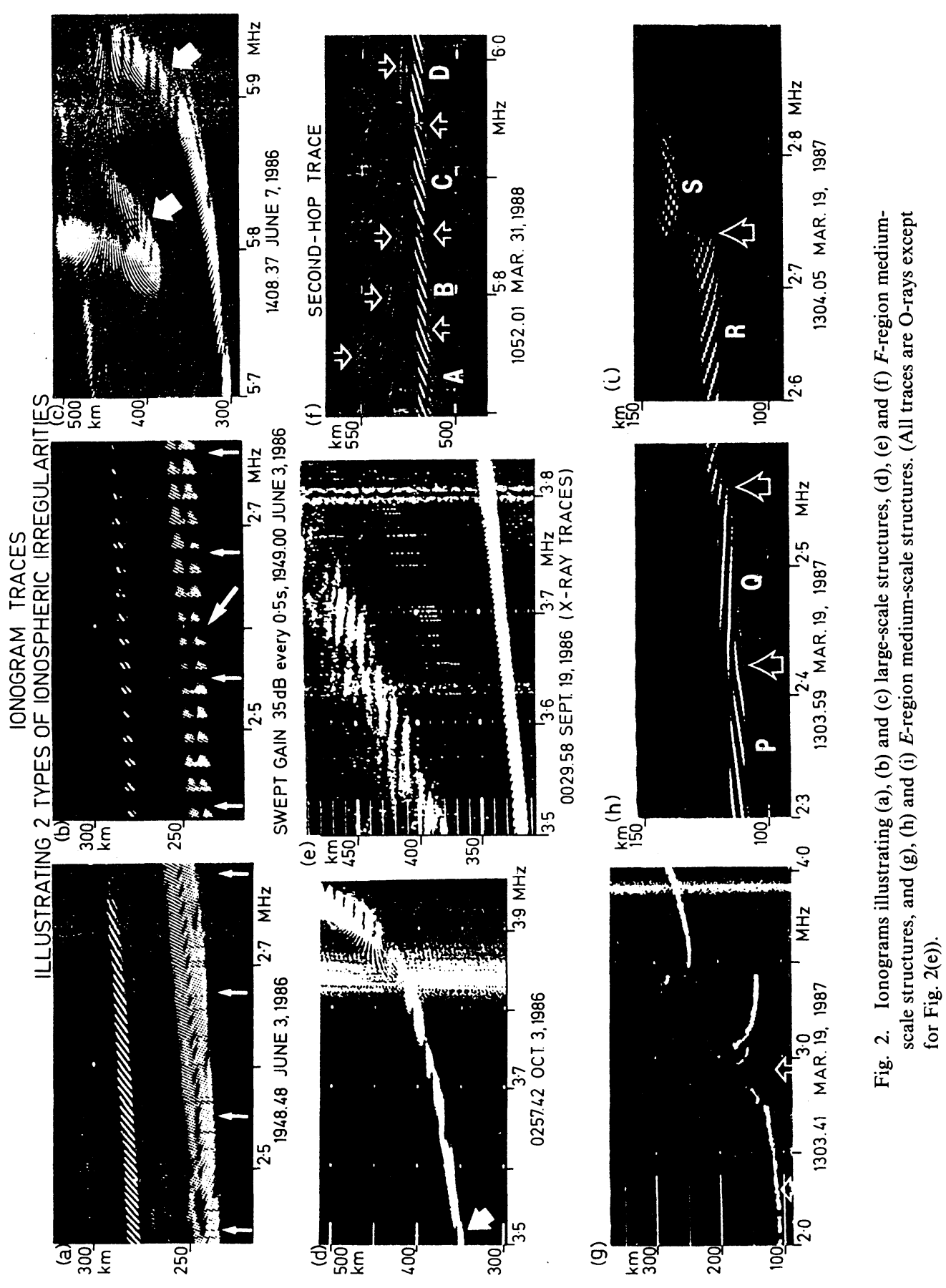

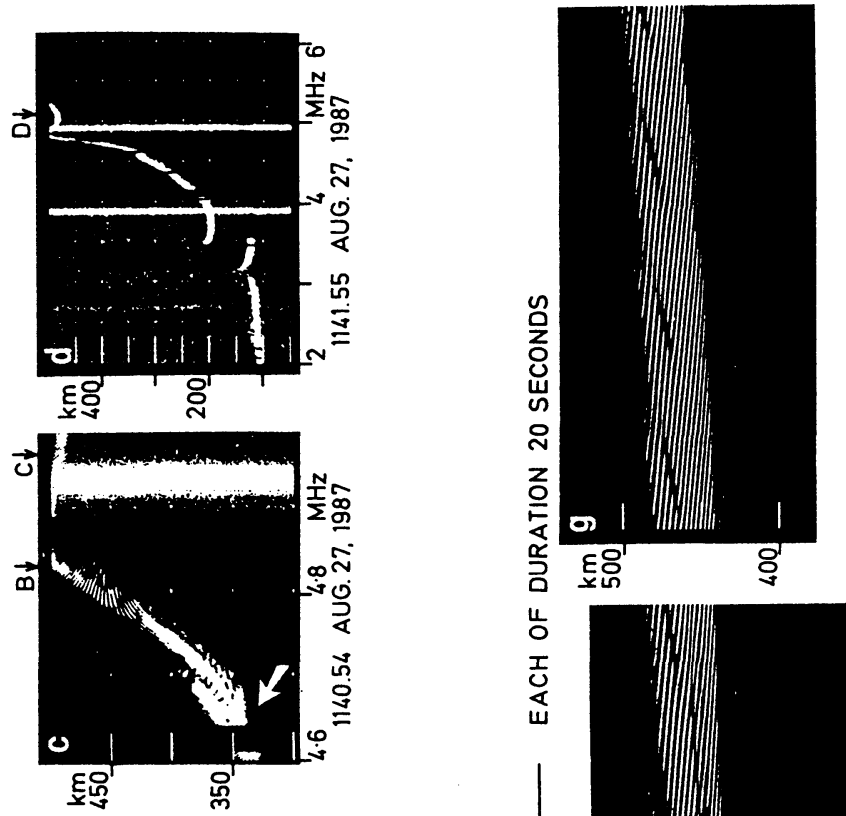

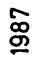

ล

एั

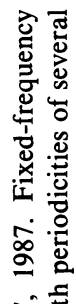

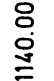

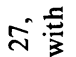

岁
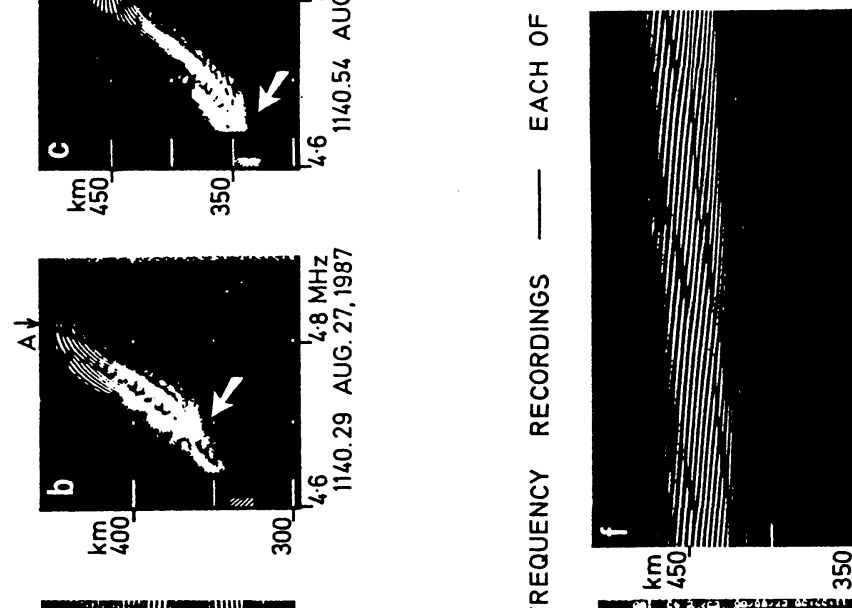

范总

ธ ธี ธั

ڤ

ก

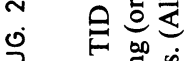

\& 苟

o 营齐嵒

잉

兽荡

동 है

(อ) क्री

ลิ
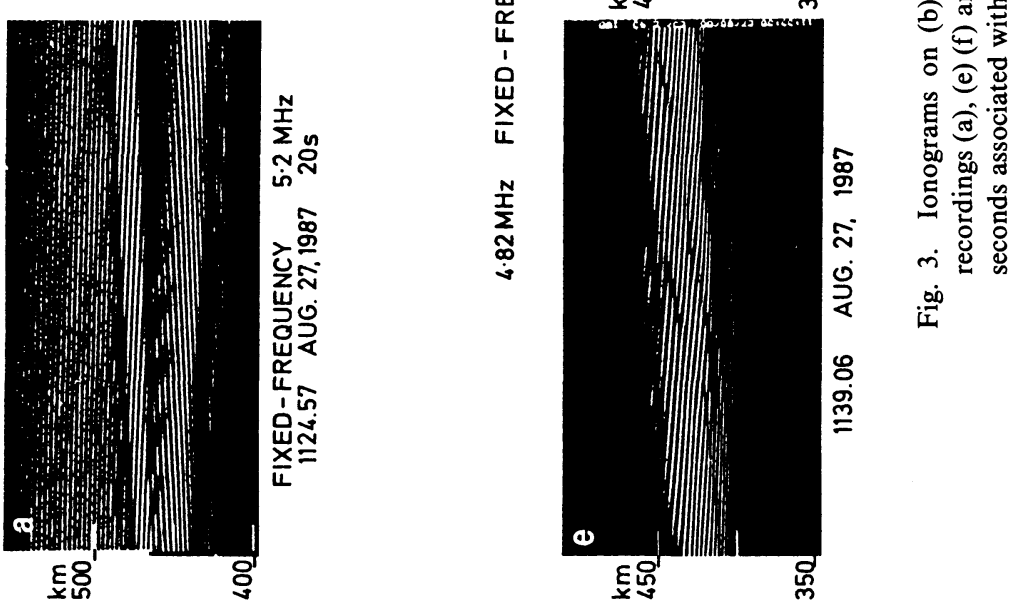
slopes of the phase fringes; the method was developed by WHITEHEAD and KANTARIZIS (1967) and explained further by BowMAN (1987). Measurements of these slopes have shown these four segments (for increasing frequency) to have virtual heights of 109.5, 113.9, 118.5 and $136.5 \mathrm{~km}$. The arrows on Figs. 2(h) and 2(i) indicate the frequencies where there are discontinuous virtual height changes at positions separating the stratifications.

Other results (also obtained recently; see BowMAN, 1987, 1988b) suggest that for both day and night hours stratification also exists at the higher levels of the ionosphere; that is, for the $F_{1}$ and $F_{2}$ layers. At these levels the main trace and the extra traces associated with TIDs can often appear broken into quasi-horizontaltrace (QHT) segments, which however are not always clearly defined, particularly during the occurrence of intense spread- $F$. Figure 1(b) illustrates these segments diagrammatically on a main trace and a duplicate trace of a nighttime ionogram. Examples of main trace segments for both day and night hours are shown by Figs. 2(c) and 2(d) respectively. The segments are more easily identified when the virtual height $\left(h^{\prime}\right)$ changes rapidly with frequency $(f)$. QHT segments associated with more complicated ionograms (e.g., when spread- $F$ is present) can be seen on ionograms illustrated by BowMAN (1987, 1988b, 1989). Figures 4(d), 4(e) and 4(f) represent such a complicated spread- $F$ ionogram. This ionogram will be discussed in more detail in the next section, where the weak signals which extend out to $4.00 \mathrm{MHz}$ will be considered. The stronger signals on Fig. 4(e) can be seen to illustrate three duplicate traces (penetrating at frequencies of $3.54,3.61$ and $3.67 \mathrm{MHz}$ ) which however are made up of QHT segments, not as easily recognizable as in Fig. 2(d).

There are times when full-range ionograms show what appear to be clean $F$-layer traces, thus giving no hint of the presence of disturbance when in fact irregularities are found to be present. However, at these times as Fig. 2(d) illustrates, QHT segments can often be seen if ionograms are recorded over limited height and frequency ranges. Even on limited-range ionograms and particularly when $\mathrm{d} h^{\prime} / \mathrm{d} f$ (the change of virtual height with frequency) is small, the main trace can at times appear clean when a disturbance is present, as Fig. 2(e) illustrates. Here (for this nighttime ionogram) the sixth-hop trace, where $\mathrm{d} h^{\prime} / \mathrm{d} f$ is significantly larger than for the first-hop trace, indicates clearly the existence of QHT segments. $N(h)$ analyses have been used to calculate a reasonable estimate of the real-height reflection levels for signals at 3.5 and $3.7 \mathrm{MHz}$. This calculation gave a separation between these levels of $4 \mathrm{~km}$, which when divided by the number of sixth-hop segments in this frequency interval gives a reasonable estimate of $0.5 \mathrm{~km}$ for the separation between stratifications. Figure 2(f) illustrates a similar method (this time for a daytime event) for detecting stratification when not suspected from the firsthop trace (not shown) of a limited-range ionogram. This figure shows that between 5.7 and 6.0 $\mathrm{MHz}$, the second-hop trace seems to consist of four QHT segments, recognized by regions of constant phase-fringe slopes, the central portions of these regions being identified by the letters $\mathrm{A}, \mathrm{B}, \mathrm{C}$ and $\mathrm{D}$. Reasonable estimates have been made of these four levels using the method involving phase-fringe slopes mentioned earlier. The virtual-height levels (for increasing frequency) were calculated to be $513.3,514.0,514.7$ and $516.3 \mathrm{~km}$. Thus it can be seen that information on what appear to be closely-packed stratifications in the lower regions of the $F_{2}$ layer can sometimes be obtained from multiple-hop traces when (even on a limited-range 

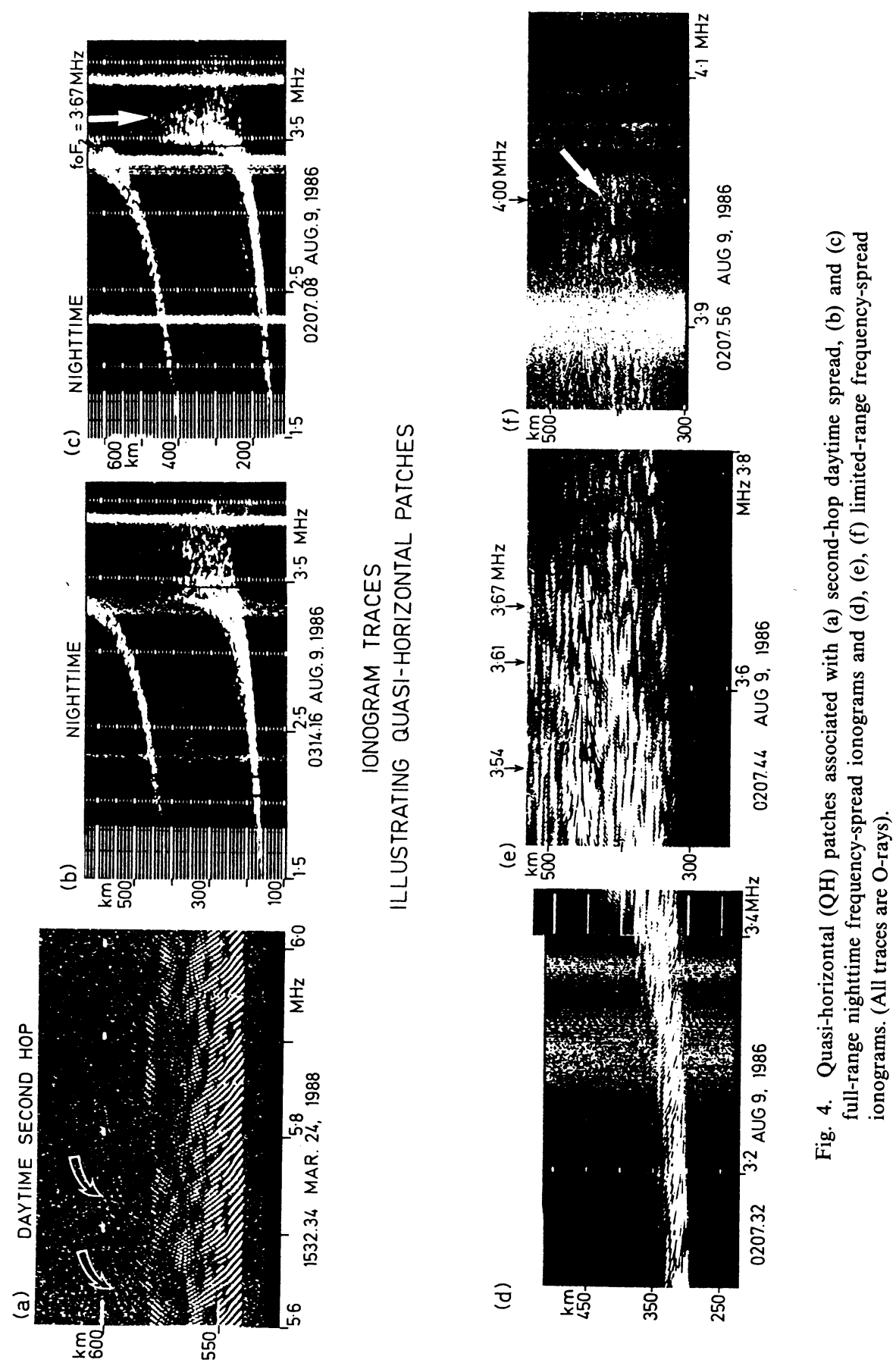
ionogram) the first-hop trace appears free of structure.

When QHT segments were present, ionograms whose recording times were separated by intervals as short as $10 \mathrm{~s}$ were found to have different patterns of segments, thus indicating the ephemeral nature of these patterns (see Figs. 5(h), 5(i) and 5(j) of BOWMAN (1988b)). The fixed-frequency recordings which have been used to investigate (second by second) these changing patterns have shown that the changes which occur are at times periodic and very systematic (BOWMAN, 1989). Some recordings have shown that as one particular segment moves out of the virtual-height range of the background ionization which reflects the small frequency range in the (so-called) fixed-frequency pulse, it is replaced by another segment. This new segment moves in from a higher or lower virtual range as the case may be. The segments are moving upwards when the background layer is rising and downwards when it is falling. The segment movements however are much faster than the background layer movements. This segment-replacement process continues to repeat itself at intervals of a few seconds. Examples of this replacement process are illustrated in Fig. 3.

Figures 3(b) and 3(c) show two limited-range ionograms spaced 25 seconds apart during the downward movement of an $f_{\mathrm{o}} F_{2}$ cusp associated with a TID. Slanting arrows illustrate the downward movement. Figure 3(d) is a full-range ionogram recorded approximately 1 minute later when this cusp is no longer observed. The arrows labelled C and D on Figs. 3(c) and 3(d) respectively point to another cusp at a high virtual range ready to move down the $F_{2}$-layer trace. Although not as clearly defined as on Fig. 2(c), QHT segments can be identified on the cusp traces of Figs. 3(b) and 3(c). Although the base of the cusp is moving down the original trace, the virtual range of the cusp trace is increasing. For example at 4.8 $\mathrm{MHz}$ the virtual range is $430 \mathrm{~km}$ at 1140.29 , while at 1140.54 it is approximately 450 $\mathrm{km}$. Prior to these times starting at 1139.06 (as shown by Fig. 3(e)) fixed-frequency recordings over a limited height range of $150 \mathrm{~km}$ were made at a frequency of 4.82 $\mathrm{MHz}$ (see arrows labelled $\mathrm{A}$ and $\mathrm{B}$ on Figs. 3(b) and 3(c) respectively). Three fixed-frequency recordings each of 20 seconds duration with starting times of 1139.06, 1139.30 and 1140.00 are shown by Figs. 3(e), 3(f) and 3(g), respectively. These figures show the upward movement of the cusp trace and more importantly, the QHT segments are seen to replace one another every few seconds by rapid upward movements of individual segments, thus illustrating the dynamic nature of these medium-scale structures. On Fig. 3(e) they are replaced every $3.9 \mathrm{~s}$ while on Fig. 3(g) the replacement rate is $3.0 \mathrm{~s}$. For comparison Fig. 3(a) shows a downward moving trace at a fixed-frequency of $5.2 \mathrm{MHz}$ (at around $425 \mathrm{~km}$ and near the $f_{\mathrm{o}} F_{2}$ value) where rapid downward movements of segments occur every $1.1 \mathrm{~s}$. A few preliminary results suggest that similar QHT segment movements occur during nighttime spread- $F$ occurrence.

\subsection{Small-scale structures}

The third class of irregularity to be discussed here is observed as weak quasihorizontal $(\mathrm{QH})$ patches. The signal strengths are some tens of decibels lower than other signals on the ionograms. These are distributed more or less randomly on the frequency-height plot of an ionogram, except that they are confined mainly to regions above the main trace and can also be found at frequencies in excess of the 
relevant critical frequency (depending on whether O-ray traces or X-ray traces are being considered). Figure 1(c) gives a diagrammatic illustration of these patches. Thus they can be distinguished as different from QHT segments, which have signal strengths comparable with other ionogram trace signals and in any case are effective subdivisions (or segments) of main and supplementary traces of ionograms. During daylight hours these patches are more often associated with second-hop traces, although these weak traces have also been recorded as constituting spreading of the first-hop daytime trace (BOWMAN and HAINSWORTH, 1987). Some additional discussion on these daytime patches can be found in BOWMAN (1988b). An example of this patchiness of second-hop daytime spread is given by Fig. 4(a). Here the spread is seen to be at least $40 \mathrm{~km}$, although (because the phase fringes allow very-weak patches to be distinguished from receiver noise) some signals (see arrows) can be seen with excess ranges as high as $60 \mathrm{~km}$. The downward pointing arrows on Fig. 2(f) also indicate very-weak patches associated with second-hop daytime spread.

To the author's best knowledge, QH patches associated with mid-latitude nighttime spread- $F$ have not been reported previously. Figures 4(d), 4(e) and 4(f) constitute a spread- $F$ ionogram (consisting of three limited-range ionograms) where these patches are present and clearly identified, extending out as far as $4.00 \mathrm{MHz}$ beyond the maximum $f_{0} F_{2}$ value of $3.67 \mathrm{MHz}$. As with the daytime patches, the signal strengths of these spread- $F$-associated patches are some tens of decibels below the strengths of the other spread- $F$ signals. Figure $4(\mathrm{c})$ is an ionogram using a frequency range of approximately $3.5 \mathrm{MHz}$ recorded shortly before Figs. 4(d), 4(e) and 4(f) which also records these patches. Figure 4(b) is another ionogram similar to Fig. 4(c), recorded approximately one hour later where these weak patches are even more obvious. It should be noted from Figs. 4(b) and 4(c) that these weak patches are not seen to be associated with the second-hop spread. As with the daytime events and illustrated by Fig. 7 of BOWMAN (1988b), it is suggested that these patches may result from normal-incidence reflection from field-aligned columns embedded in the stratified regions suggested by the QHT segments.

The presence of $\mathrm{QH}$ patches on ionograms can be determined by identifying the associated straight under edges as illustrated by Figs. 4(e) and 4(f) as they extend beyond the O-ray or X-ray critical frequency of an ionogram. These patches are most likely to be recorded for a period around the June solstice (for both hemispheres-BOWMAN, 1964) of sunspot-minimum years when mid-latitude spread- $F$ activity is most intense (BOWMAN, 1960a) and present almost every night. These weak echoes extending out beyond $f_{0} F_{2}$ (for O-rays) are similar to the socalled "slant- $F$ " phenomenon reported by BATES $(1959,1971)$ for auroral-zone regions. These auroral-zone echoes were found to be aspect-sensitive, thus suggesting that field-aligned irregularities (FAI) were involved (BATES, 1971; BATES and ALBEE, 1970).

\subsection{Macroscale structures}

A well-known macroscale structure change associated with spread- $F$ occurrence is the large ionospheric height rise which occurs prior to the occurrence of postsunset spread- $F$ in equatorial regions (SKINNER and KELLEHER, 1971; RASTOGI, 1981). However, what is less well known is the fact that similar (although not nearly 
as pronounced) height rises occur with associated spread- $F$, at other latitude regions (MCNicOL et al., 1956; BowMAN, 1960b, 1978). Even in high-latitude regions spread- $F$ occurrence is found to be related to higher $h^{\prime} F$ and lower $f_{0} F_{2}$ values (see Fig. 15 of HERMAN, 1966; also SHIMAZAKI, 1962).

In recent times spread- $F$ related height rises in mid-latitude regions have been found to be modulated by oscillations in the isoionic contours with periodicities around 20 minutes. These have been detected by BOWMAN (1986) and BOWMAN and MONRO (1988) using $N(h)$ analyses. Similar modulations have also been reported by FROM and MEEHAN (1988) using doppler-shift measurements by a modern HF radar. Furthermore, STERLING et al. (1971) have reported wavelike perturbations (periodicity approximately 20 minutes) in the isoionic contours during the postsunset height rises which precede equatorial spread- $F$ occurrence. This paper will consider not only macroscale structure changes as they apply to medium-scale TIDs which MCNiCOL et al. (1956) associated with mid-latitude spread-F occurrence, but also as they apply to large-scale TIDs (FRANCIS, 1975) which also at times (see e.g., BOWMAN, 1978) appear to be associated with spread- $F$ occurrence.

\subsubsection{Large-scale TIDs}

A review by FRANCIS (1975) has summarized the evidence for large-scale TIDs. FRANCIS (1975) states "Large-scale TIDs generally travel from the polar regions towards the Equator with speeds between 400 and $1000 \mathrm{~m} / \mathrm{sec}$ ". More recently such movements have been reported by HAJKOWICZ (1983) and HAJKOWICZ and HUNSUCKER (1987), by considering the times of onset of the large height rises which accompany these disturbances. Figure 6 of TANAKA (1987) shows the progression of these height rises to lower latitudes, as the disturbance moves towards the equator. Gigahertz scintillations are also involved at these times. It has been shown that on occasions the height rise is preceded by a brief period when the ionospheric level falls (see Fig. 7 of BowmAN (1965)), where also the recordings show that the $f_{\mathrm{o}} F_{2}$ value is reduced considerably at the time of the height rise.

Of particular interest to this paper is the fact that on some occasions (but not always) spread- $F$ is recorded during and for some time after the height rise. Evidence for this associated spread- $F$ occurrence is presented in a number of papers (e.g., BowMAN, 1965, 1978; BOWMAN and SHRESTHA, 1966). Large-scale TIDs have also been detected in the ionosphere following nuclear explosions where height rises, $f_{\mathrm{o}} F_{2}$ reductions and spread $F$ are also recorded (see Plates 1 and 2 of BOWMAN, 1962).

Figure 5 illustrates some of the ionograms associated with the passage of a large-scale TID at four Australian ionosonde stations on May 15, 1969. The geomagnetic coordinates for these stations (Canberra, Norfolk Island, Brisbane and Townsville) are given by HAJKOWICZ and HUNSUCKER (1987). Spread- $F$ is seen only on the Canberra ionograms. The isoionic contours obtained by $N(h)$ analyses (SCHMERLING, 1967) of the Brisbane ionograms of this event are shown by Fig. 1 of BOWMAN (1977). The macroscale structure changes (small height reduction, large height rise and maximum plasma frequency reduction from about $6.0 \mathrm{MHz}$ to about $4.8 \mathrm{MHz}$ ) are shown clearly by this figure. The disturbance onset time delays between stations for this event give an approximate velocity of $308 \mathrm{~ms}^{-1}$ in the direction $5^{\circ}$ east of true north.

The ionospheric tilts associated with large-scale TIDs can be expected to be 


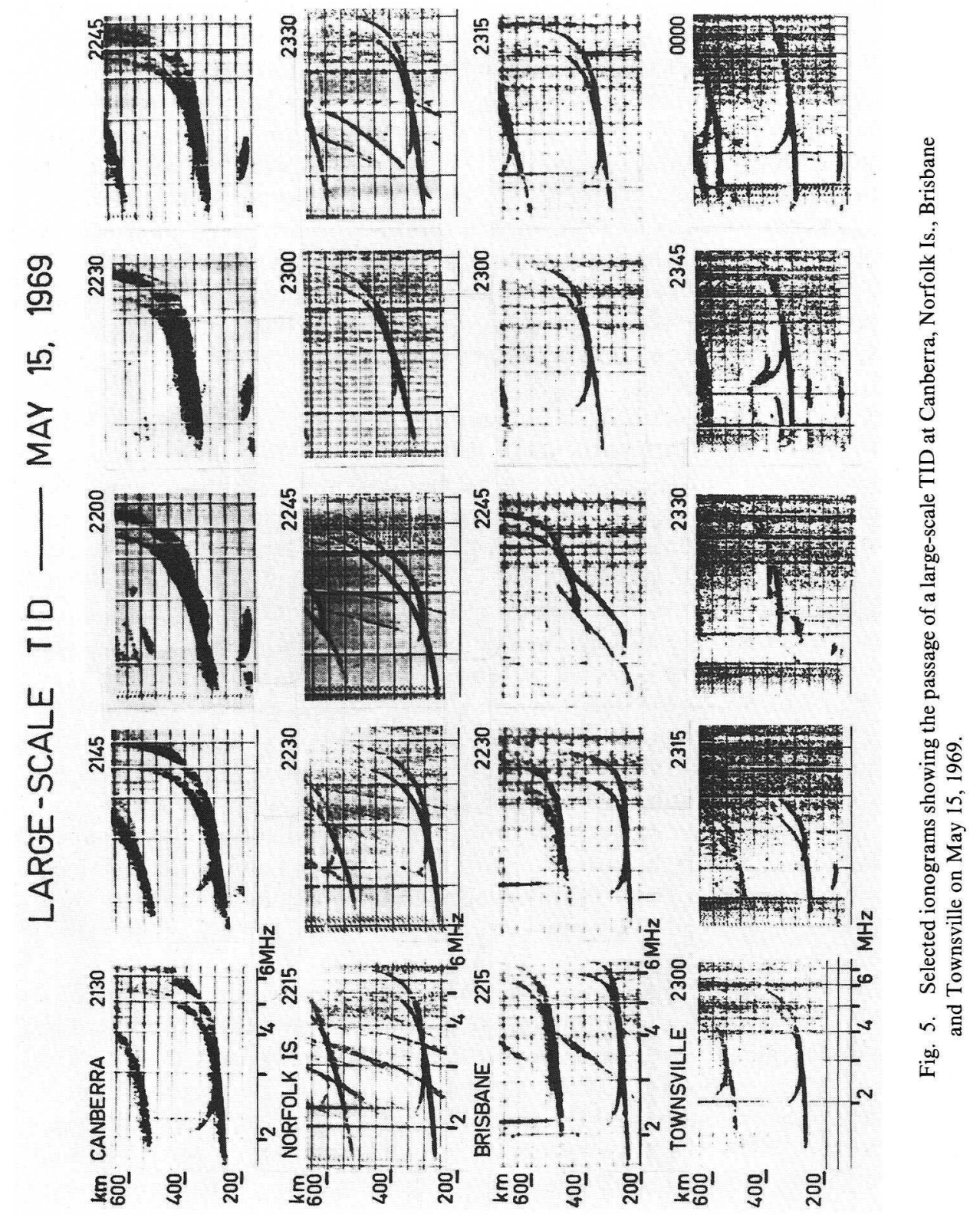


significantly smaller than those associated with medium-scale TIDs, since there is approximately an order of magnitude difference in their speeds. This is illustrated by direction of arrival measurements by CLARKE (1972). For the passage of a largescale TID over Bribie Island on November 19, 1970, he found the layer tilted by only 2 degrees during a half-hour period when the layer height changed by $100 \mathrm{~km}$. This tilt should be compared with that for a medium-scale TID (speed $40.5 \mathrm{~ms}^{-1}$ ) at Bribie Island on January 15, 1986, where the tilt is approximately 17 degrees (see Fig. 6 of BOWMAN and MONRO, 1988) for an overall height rise of $50 \mathrm{~km}$ in approximately 1 hour. The fact that spread- $F$ is not always associated with large-scale TIDs may possibly be explained by the smallness of the associated tilt.

What appears to be another important aspect of large-scale TID characteristics has been reported by OYA et al. (1982). Their paper has shown that satellite recordings indicate "wavy patterns of ionospheric electron density" recorded at times when large-scale TIDs are expected as judged from substorm onset times (BOWMAN, 1978; TAKAHASHI et al., 1987). Group velocities in the range $250 \mathrm{~ms}^{-1}$ to $760 \mathrm{~ms}^{-1}$ were found by OYA et al. (1982) for the TIDs and the average period for the wavy patterns was calculated at 16.7 minutes. These periodicities should be compared with those to be reported for medium-scale TIDs in the next subsection (see Table 1). Thus it seems likely that a periodic oscillation (average period $16.7 \mathrm{~m}$ ) modulates the macroscale height rises, which occur over periods sometimes in excess of 30 minutes during the passage of large-scale TIDs.

\subsubsection{Medium-scale TIDs}

The simplest form of mid-latitude spread- $F$ as seen on ionograms consists of a single duplicate trace which usually moves either towards or away from the main trace. These movements can be recorded continuously using fixed-frequency transmissions, which result in (so-called) $h^{\prime} t$ recordings (i.e., recordings of virtual height versus time). MCNICOL et al. (1956) classified these duplicate traces as either parallel, convergent, divergent or mixed, depending on the variation of their range with time on $h^{\prime} t$ recordings. The relative frequency of occurrence of these different classifications were "roughly in the ratio 1:8:20:6". Thus it can be seen that divergent traces (which are most prevalent) are recorded 2.5 times more often than convergent traces. Plate 5 of MCNicOL et al. (1956) illustrated isolated duplicate trace movements. Their Plate 3 shows examples of the mixed type, when the spread- $F$ phenomenon is more complicated, consisting of a series of duplicate traces which sometimes overlap each other, making it difficult to identify separate duplicate traces.

Some of the recordings made for the investigation by MCNICOL et al. (1956) are presented in Fig. 6 to illustrate the height rises associated with this mid-latitude spread- $F$ occurrence. On the night of October $9 / 10,1952$, the $h^{\prime} t$ recordings show four spread- $F$ events starting at times of 19:00, 21:30, 23:30, and 02:30; each event lasts for approximately one hour. Two of these events (starting times 21:30 and 23:30) are shown on Figs. 6(a), 6(b) and 6(c) (four hours of recording) where diverging duplicate traces are preceded by height rises, the extent of which (in each case) is indicated on the diagrams. Figures 6(d) and 6(e) show a mixed event on August 6, 1951, showing converging duplicate traces before the maximum height (reached at around 19:50) to be followed by diverging duplicate traces. Early investigators recognized the single converging trace (similar to that shown by Fig. 


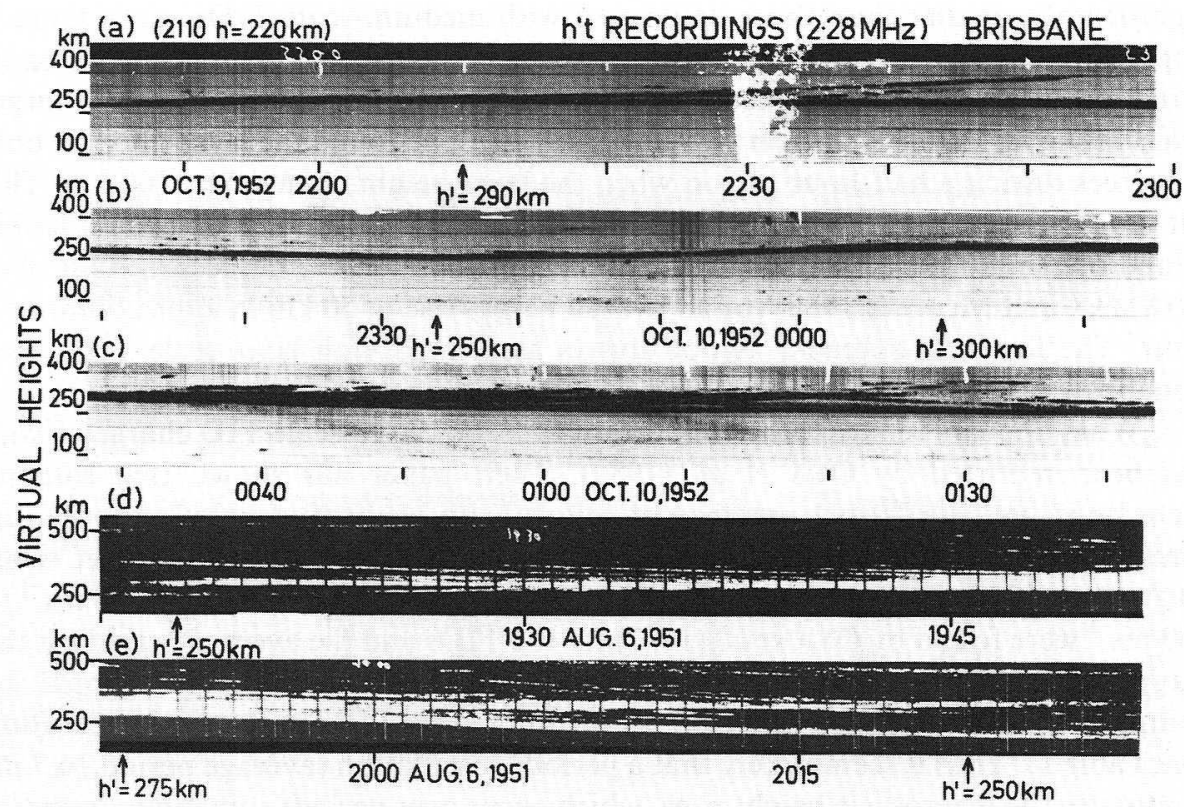

Fig. 6. Fixed frequency $(2.28 \mathrm{MHz}) h^{\prime} t$ recordings for Brisbane showing; (a), (b) and (c) height rises and diverging duplicate traces on October 9/10, 1952, and (d) and (e) a height rise with converging and diverging duplicate traces on August 6, 1951.

6(d)) which sometimes appears before the more complex spread- $F$ structures. In a review paper, HERMAN (1966) discusses this converging trace under the heading "the traveling reflection". The events shown by Fig. 6 are typical of mid-latitude spread- $F$ occurrence as recorded at fixed frequency $(2.28 \mathrm{MHz})$. The investigation by MCNICOL et al. (1956) provided strong evidence for associating most spread- $F$ seen at mid-latitudes with medium-scale TIDs. Using three spaced stations (approximately $100 \mathrm{~km}$ apart) they were able to show that the spread- $F$ traces resulted from the passage of frontal disturbances with speeds in the range 42 to $97 \mathrm{~ms}^{-1}$.

Several $N(h)$ analyses (SCHMERLING, 1967) have been performed to investigate the wavy patterns which were found previously (BOWMAN, 1986; BOWMAN and MONRO, 1988) superimposed on the macroscale height change preceding spread- $F$ occurrence. Figure 7 shows some of the disonde ionograms for a spread- $F$ event on the night of October $8 / 9,1988$. A height rise followed by diffuse spread- $F$ traces (some structure is evident-see arrows) is shown. Some (weak) X-ray traces can also be seen. Figure $8(\mathrm{~b})$ shows isoionic contours produced by the $N(h)$ analyses for this event, until the end of recording at 01:27.05. For 2 hours prior to the start of the height rise at around 00:30, the distribution of ionization remained relatively constant except that the $f_{\mathrm{o}} F_{2}$ gradually decreased, as illustrated by the line $\mathrm{AB}$ on Fig. 8(a). During the recorded height rise from 00:30 to 01:27, where there is some evidence of an oscillation with a period of around 15 minutes, there is a sudden change in the gradient of $f_{\mathrm{o}} F_{2}$ as illustrated by line $\mathrm{CD}$ on Fig. 8(a). The extent of the 

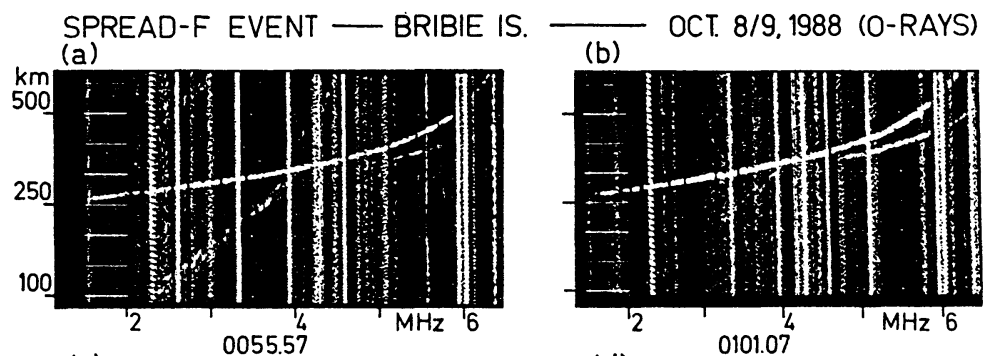

(c)
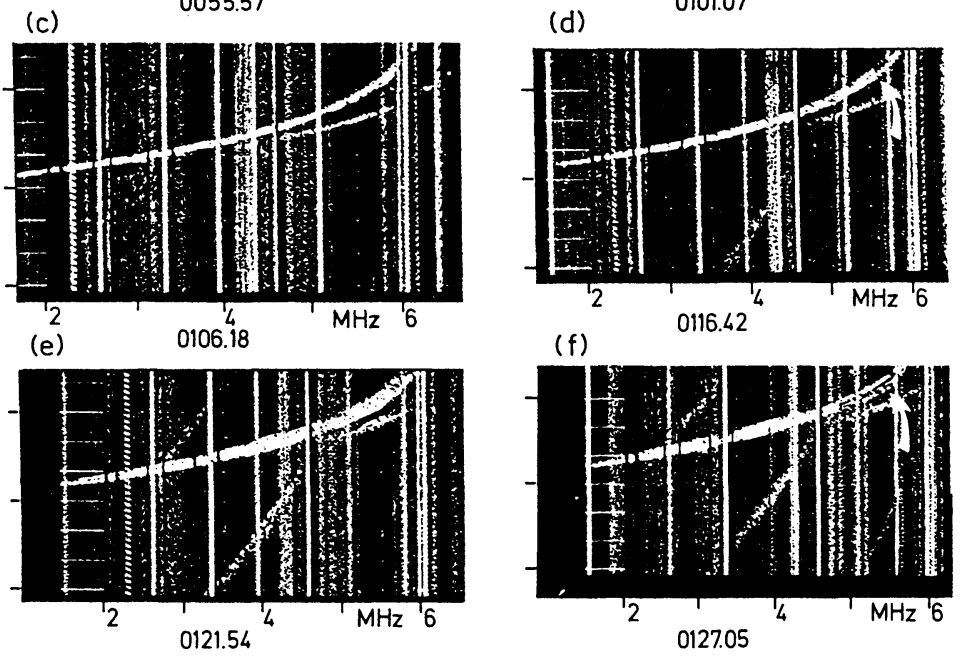

Fig. 7. Selected ionograms for spread- $F$ event at Bribie Island on October 8/9, 1988.

range spread (following the commencement of the height rise) starting at 01:01 (approximately one half hour after the initial height rise) is shown on Fig. 8(c). Figure 9 illustrates another spread- $F$ event (October $1 / 2,1988$ ) showing an $f_{\mathrm{o}} F_{2}$ reduction (of $0.5 \mathrm{MHz}$ ), follow by recovery and an oscillatory pattern (periodicity around 12 minutes) associated with the height rise.

Figure 10 shows the $f_{\mathrm{o}} F_{2}$ variation (Fig. 10(a)) and the isoionic contour variations (Fig. 10(b)) of a spread- $F$ event on October 16, 1988, which produced a mixed (single duplicate trace) event as illustrated by Figs. 11(a)-11(f). On Figs. 11(c) and $11(\mathrm{~d})$ parts of additional traces are seen (see downward pointing arrows) while the section of trace indicated by the upward pointing arrow is a weak X-ray trace. A wavetrain with a periodicity of 13 minutes is suggested by Fig. 10(b). It seems likely that the duplicate trace in Fig. 11(a) (recorded at position A in Fig. 10(b)) is a converging trace, while the duplicate traces in Figs. 11(c)-11(f) are obviously diverging and recorded at times indicated by letters $\mathrm{C}-\mathrm{F}$ in Fig. 10(b). The duplicate trace in Fig. 11(b) is barely perceptible and could be either converging or diverging (see position B on Fig. 10(b)). The analysis presented to this point has considered events where the spread- $F$ occurrence has been mild or of simple form (e.g., the single converging and diverging traces in Figs. 11(a)-11(f)). More extensive (and 


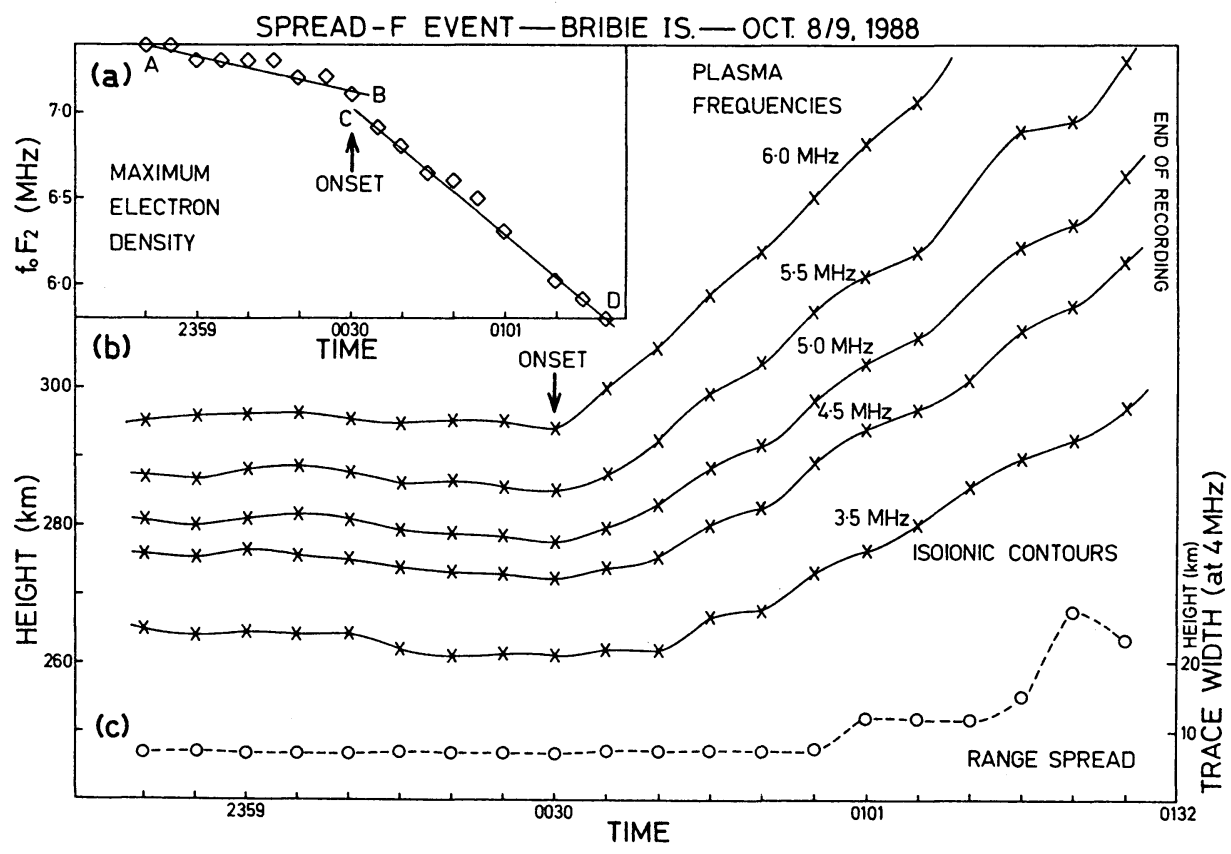

Fig. 8. (a) $f_{0} F_{2}$ variations, (b) variations in isoionic contours and (c) width of $F_{2}$ region trace (range spread) for spread- $F$ event on October $8 / 9,1988$.

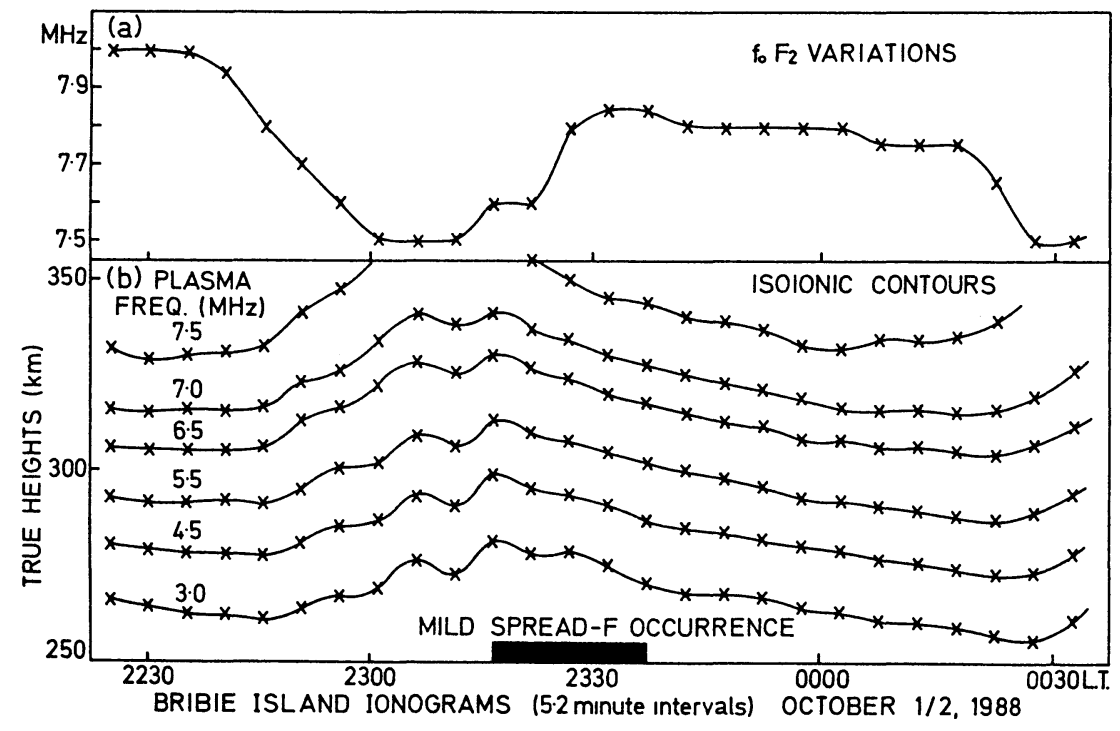

Fig. 9. (a) $f_{\mathrm{o}} F_{2}$ variations and (b) variations in isoionic contours for event on October $1 / 2,1988$. 


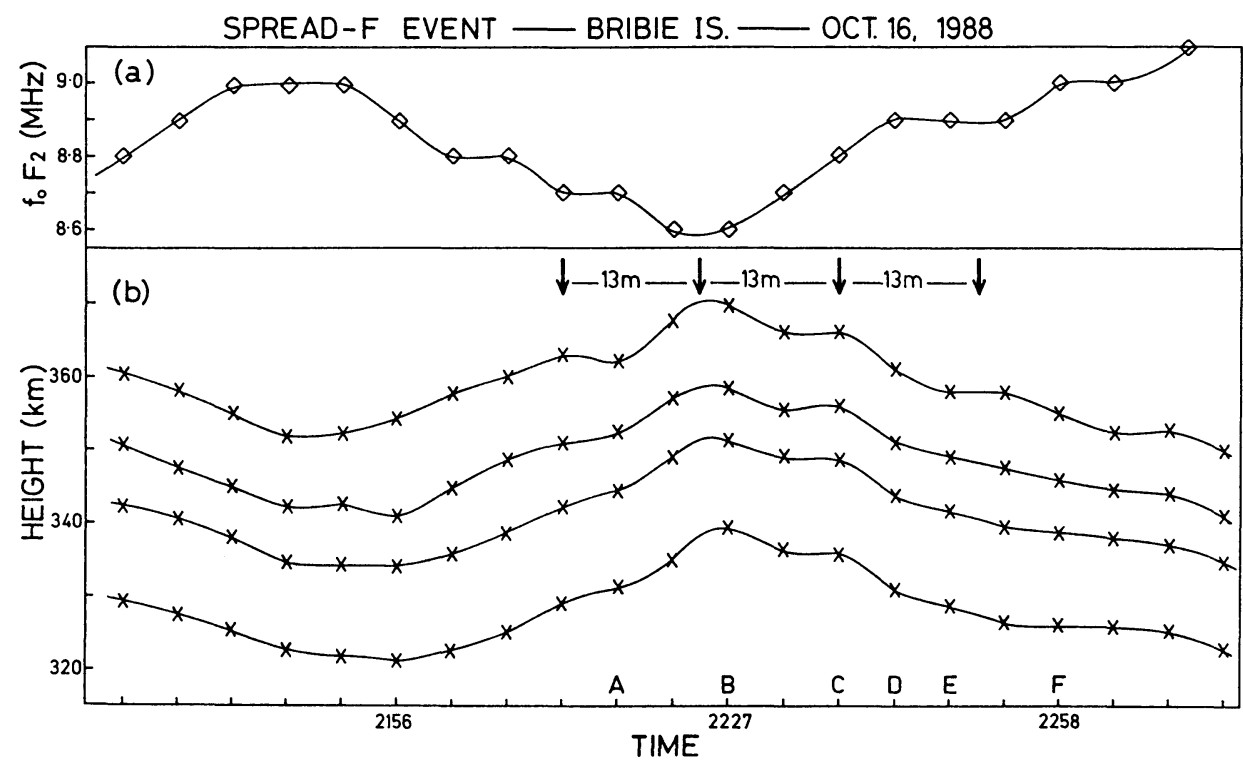

Fig. 10. (a) $f_{\mathrm{o}} F_{2}$ variations and (b) variations in isoionic contours for event on October 16, 1988.

complicated) spread- $F$ forms will now be considered. Also, due to limited recording periods of the disonde, as well as the fact that the disonde recorded X-rays only on some occasions, some $N(h)$ analyses have used Moggill ionograms (at 15 minute intervals) where oscillatory patterns are not expected to be recorded due to the spacing between ionograms.

Range spread as large as $80 \mathrm{~km}$ is evident for a spread- $F$ event on June 20,1988 , where (as shown in Figs. 11(g)-11(j)) nevertheless much structure (in the form of duplicate traces) is in evidence. Figure 12(a) (which uses Moggill 15 min ionograms) shows the associated height rise which starts abruptly at around 19:00, while Fig. 12(b) illustrates the associated $f_{\mathrm{o}} F_{2}$ variations and the extent of the excess range spread recorded. The times of recording of the four ionograms in Figs. 11(g)-11(j) are indicated on Fig. 12 by the letters G-J. Figures 11(k) and 11(l) illustrate the extreme spread- $F$ conditions which are sometimes present in mid-latitude regions. These particular spread- $F$ ionograms were recorded on the night of July $2 / 3,1988$. Range spreads in excess of $100 \mathrm{~km}$ are recorded at various times throughout the night. These ionograms are very diffuse making it difficult to identify duplicate traces. Furthermore, the degree of spread is so extreme that it is impossible to read $f_{\mathrm{o}} F_{2}$ values with any certainty. Although on July $2 / 3,1988$ spread- $F$ was present all night, Fig. 13(a) (which uses Moggill ionograms) indicates that on four occasions during the night height rises were accompanied by increased range spread, thus suggesting a succession of four spread- $F$ events merged into each other, giving spread- $F$ on ionograms all night. The recording times for Figs. 11(k) and 11(l) are indicated by the letters $\mathrm{K}$ and L on Fig 13(a). The scale in Fig. 13(a) applies to both the $h^{\prime} F$ and range-spread values, as the range-spread positions indicate the maximum height extent (at about $3 \mathrm{MHz}$ ) of the spread. A reasonable estimate of 

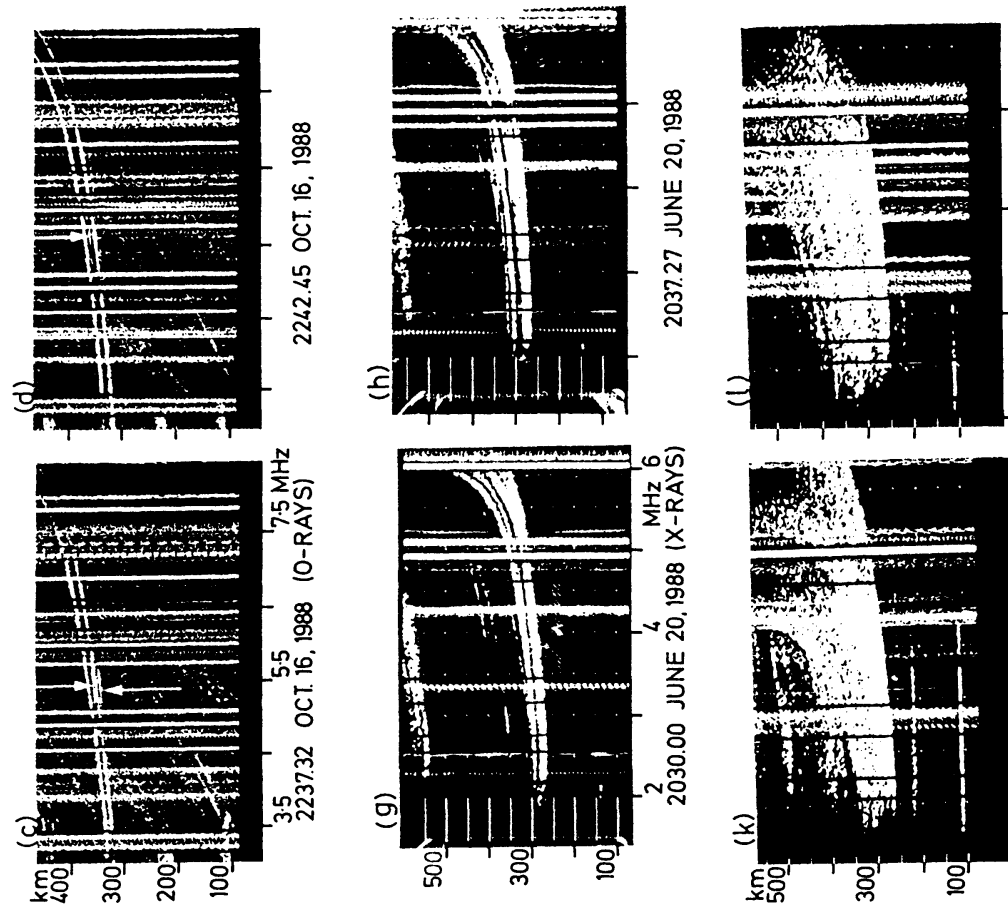

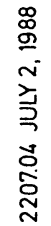

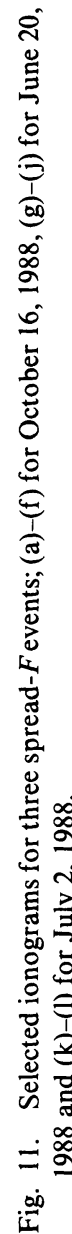
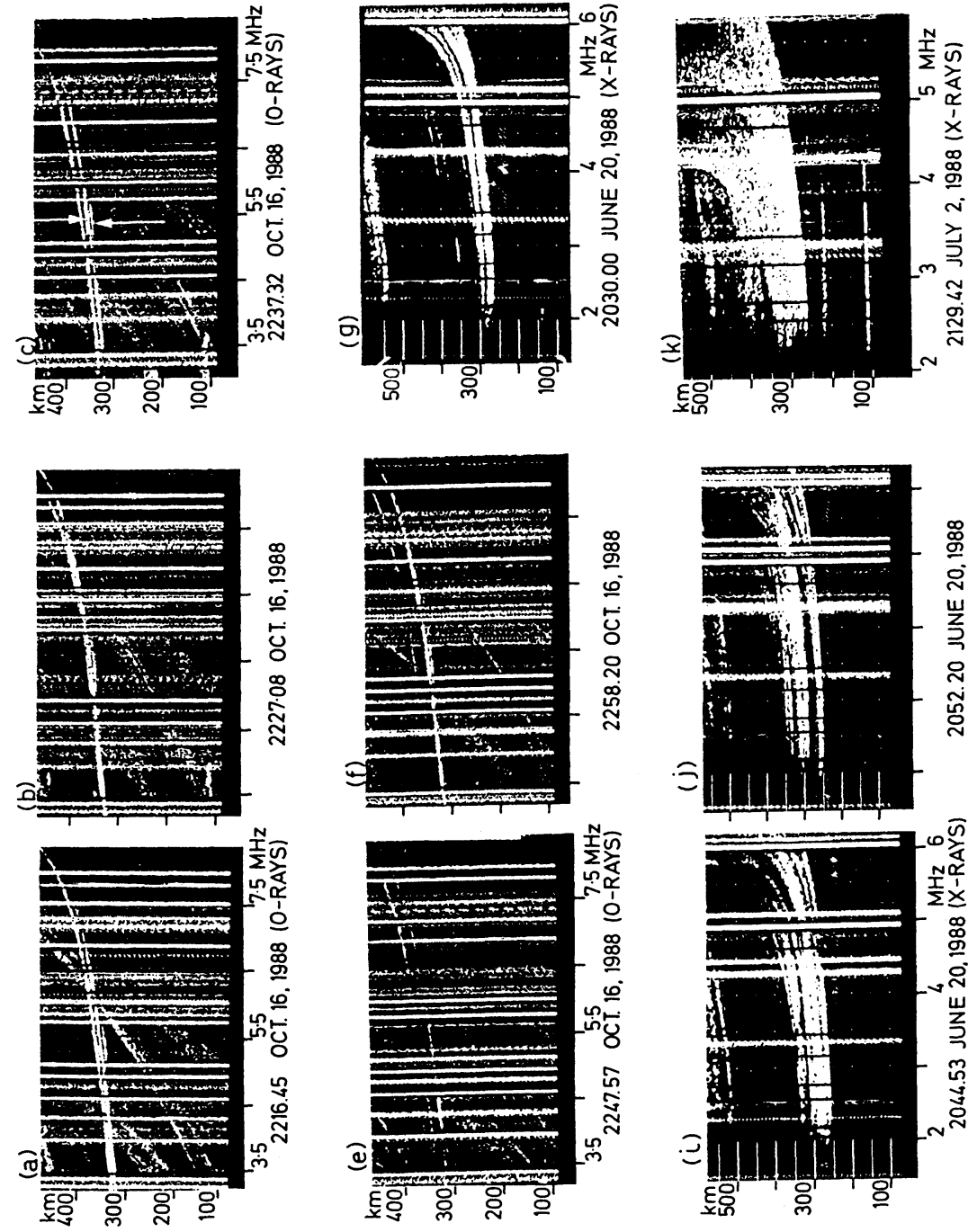


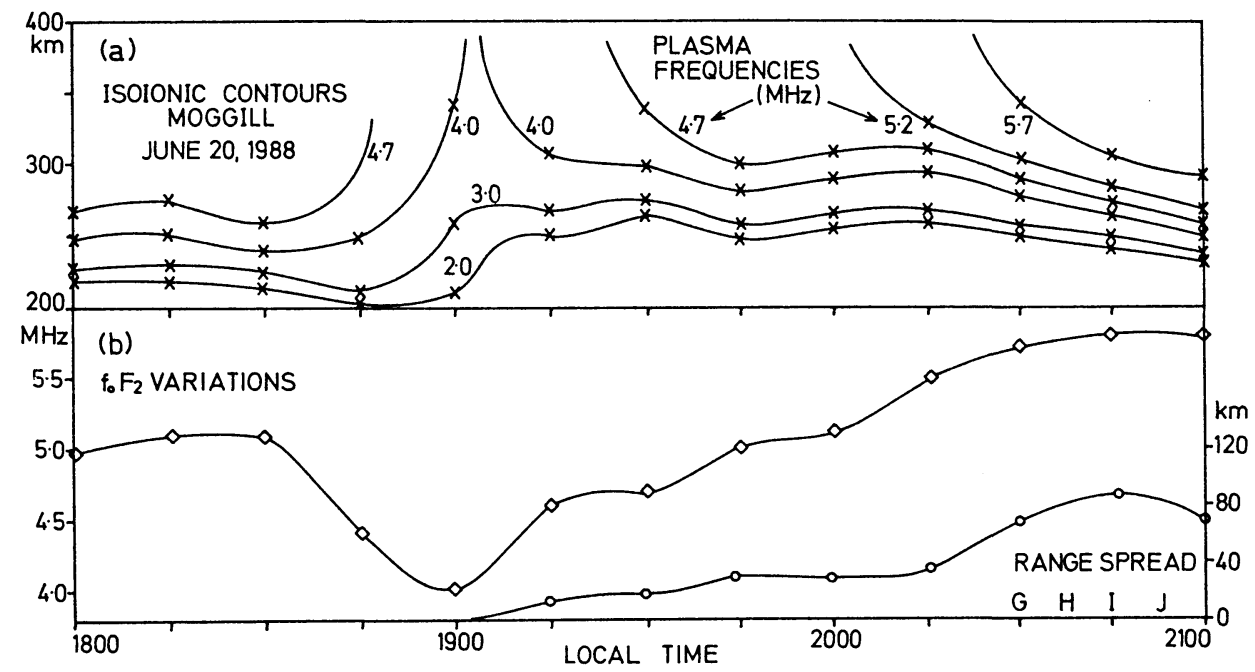

Fig. 12. (a) Isoionic contours (Moggill ionograms) and (b) $f_{\mathrm{o}} F_{2}$ variations and excess range spread, for spread- $F$ event on June 20, 1988.

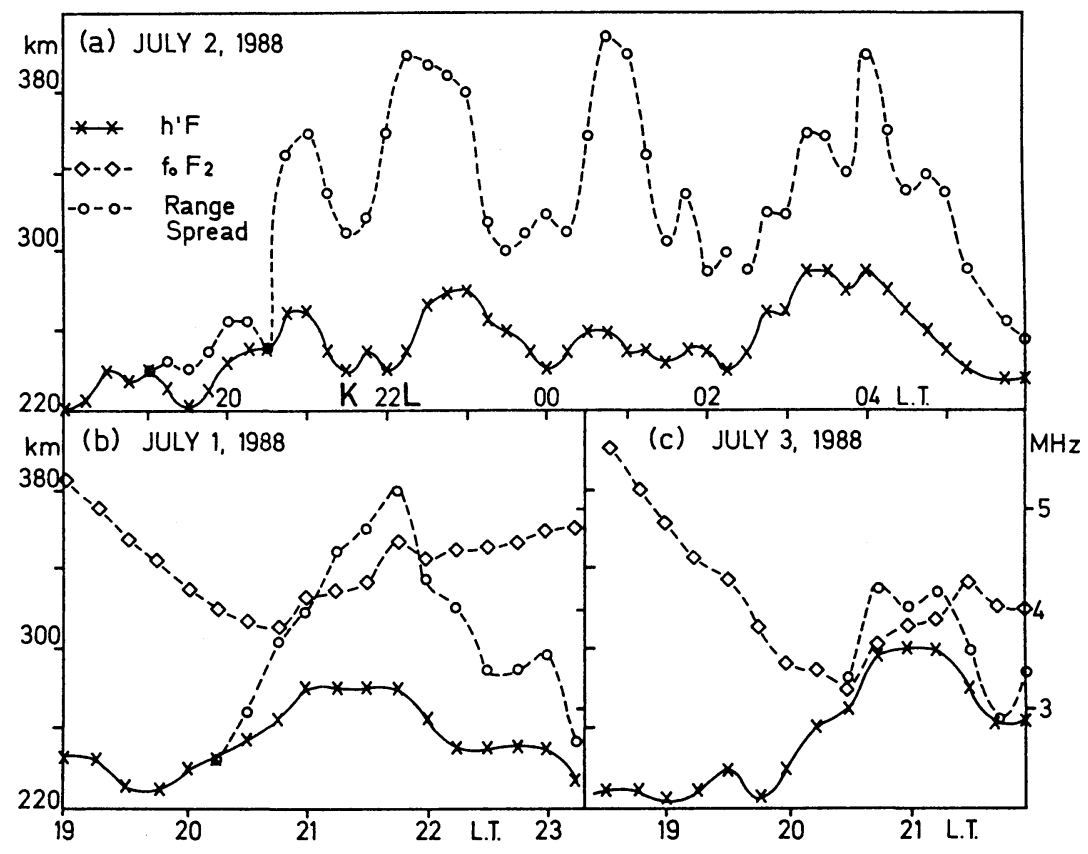

Fig. 13. Variations in $h^{\prime} F, f_{\mathrm{o}} F_{2}$ and range spread (Moggill ionograms) for spread- $F$ events on (a) July 2, 1988, (b) July 1, 1988 and (c) July 3, 1988. 
the spread can be obtained at any time by subtracting the $h^{\prime} F$ value from the maximum range-spread height. An early paper by BowMAN (1960b) (see his Fig. 3) shows similar effects between the degree of range spread and height rises. Figures 13(b), 13(c) and 14 show further examples (using Moggill ionograms) of this relationship between height rises, $f_{0} F_{2}$ reductions and spread- $F$ occurrence. Thus it can be seen that it is not difficult in mid-latitude regions to identify macroscale changes (involving height and electron-density changes) with large-scale periodic wavelike structures and subsequent spread- $F$ occurrence. It is convenient to regard all these changes (considered as a whole) as a "spread- $F$ event".

Table 1 summarizes the results of 6 different analyses, each of which suggest that TID wavetrains modulate the isoionic contours during macroscale height rises.

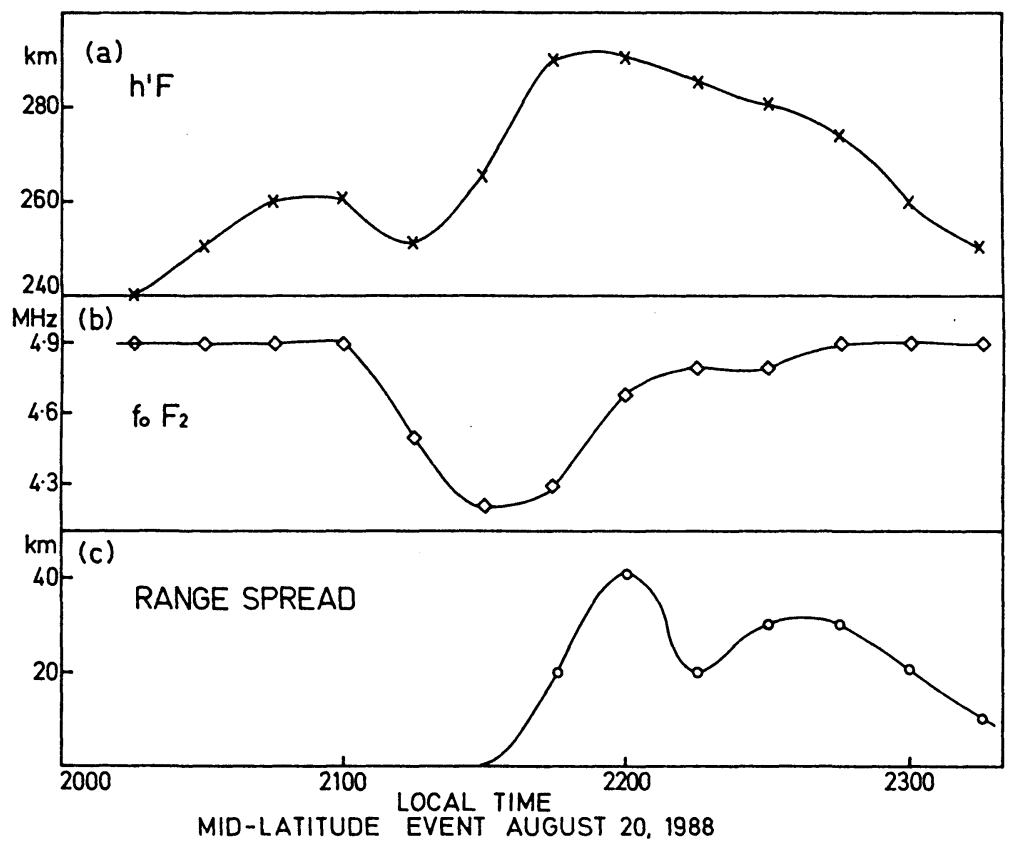

Fig. 14. Variations in (a) $h^{\prime} F$, (b) $f_{\mathrm{o}} F_{2}$ and (c) range spread (Moggill ionograms) for spread- $F$ event on August 20, 1988.

Table 1. Wavetrains detected during spread- $F$ events.

\begin{tabular}{llccl}
\hline \multicolumn{1}{c}{ Date } & Method & $\begin{array}{c}\text { Period } \\
(\mathrm{mins})\end{array}$ & $\begin{array}{c}\text { Wavelength } \\
(\mathrm{km})\end{array}$ & \multicolumn{1}{c}{ Reference } \\
\hline May 30, 1980 & $N(h)$ analysis & 28 & 65 & Bowman (1986) \\
Jan. 15, 1986 & $N(h)$ analysis & 20 & 50 & BowmAN and MoNRo (1988) \\
Oct. 8/9, 1988 & $N(h)$ analysis & 15 & & This paper (Fig. 4) \\
Oct. 1/2, 1988 & $N(h)$ analysis & 12 & & This paper (Fig. 5) \\
Oct. 16, 1988 & $N(h)$ analysis & 13 & & This paper (Fig. 6) \\
Aug. 27, 1981 & Doppler shift & 24 & & From and MEEHAN (1988) \\
\hline
\end{tabular}


Various periodicities are found but the average value is 19 minutes. Five of these results were achieved by $N(h)$ analyses. The sixth result, reported recently by FROM and MEEHAN (1988) (see their Figs. 2(a) and 2(b)), was obtained from the recordings of a modern HF radar. A periodicity of around 24 minutes starting at about 21:00 at the time of commencement of the height rise is detected primarily by doppler shift measurements. However, it can also be seen in the accurate virtual height measurements (their Fig. 2(b)). Off-vertical spread- $F$ signals were first detected at around $21: 35$ on this occasion.

\section{Comparisons of Spread-F Events with Other Phenomena}

Although this paper is primarily concerned with mid-latitude spread- $F$ events, some time will be spent briefly considering spread- $F$ results from other latitude regions, so that comparisons can be made with the mid-latitude results. In particular this section will consider the salient features of (a) post-sunset equatorial spread- $F$ (ESF), (b) mid-latitude ionospheric troughs, and (c) ionospheric changes associated with the occurrence of stable auroral red arcs (SAR arcs). To avoid confusion with the mid-latitude spread- $F$ region discussed in this paper, which is somewhat removed from the trough region, the trough will hereafter be referred to as the sub-auroral ionospheric trough (SAI trough).

For some time now experimental evidence has been accumulating suggesting a possible relationship between equatorial spread- $F$ and atmospheric gravity waves (AGWs) (ROTTGER, 1973; TsUNODA, 1981, 1983; KELley et al., 1981; ARGO and KELLEY, 1986). In the post-sunset height rise associated with spread- $F$ occurrence the (so-called) "west walls upwellings" are found to contain wavelike modulations similar to that shown by Fig. 15 (see Fig. 10 of TSUNODA et al., 1982). These large-scale structures move to the west with speeds which suggest they may be

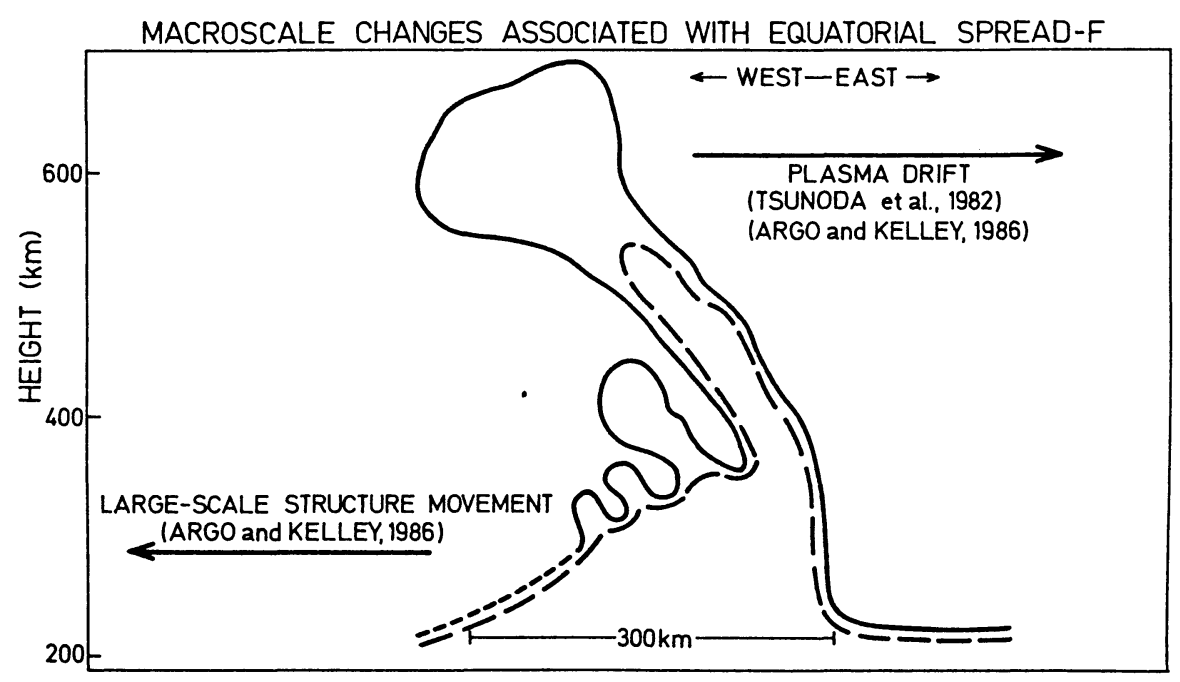

Fig. 15. Macroscale changes associated with equatorial spread-F (after TsunODA et al., 1982). 
related to AGWs generated by the terminator (CLARK and RAITT, 1976). The spread- $F$ on ionograms is recorded as the layer falls (ARGO and KELLEY, 1986) similar to most of the mid-latitude results. This is what would be expected if (as shown for mid-latitude regions-BOWMAN and MONRO, 1988) the wavelike structures must move away from the overhead position, before the excess ranges for signals reflected from them are sufficiently large for the spread traces to be observed on ionograms. As Fig. 15 shows, the sequence of events for this post-sunset ESF is very similar to that found for mid-latitude spread- $F$, where, however, the height rises are generally much smaller. Also the large-scale plumes present for equatorial regions are not seen at mid-latitudes. STERLING et al. (1971) have reported wavelike perturbations (periodicity approximately 20 minutes) in the isoionic contours during post-sunset height rises in an equatorial region (see their Fig. 2). Furthermore, for the specific events reported by STERLING et al. (1971), the published hourly values of ionospheric parameters show that these particular height rises are followed by spread- $F$ occurrence. Also, ALEX et al. (1989) have shown recently that post-sunset equatorial $f_{\mathrm{o}} F_{2}$ values are much lower on nights when spread- $F$ follows a significant height rise than on other nights. Macroscale equatorial spread- $F$ structures moving with speeds comparable with those of medium-scale TIDs are found at times away from the equatorial post-sunset period. For example, the fixed-frequency recording shown by Fig. 3 of WEBER et al. (1978) is very similar to Figs. 6(d) and 6(e) of this paper. Other similarities of spread- $F$ characteristics between these two regions are reported by BOWMAN (1984).

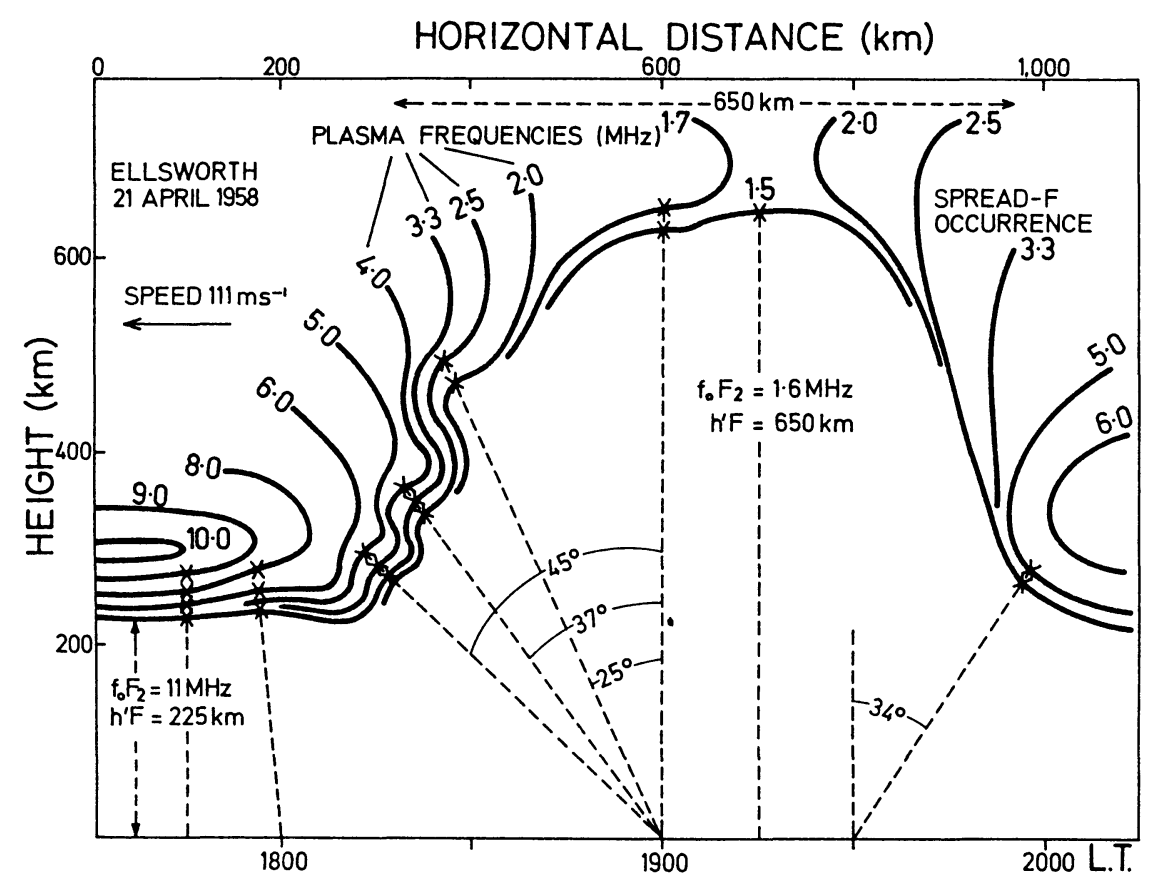

Fig. 16. Example of sub-auroral ionospheric trough at Ellsworth on April 21, 1958 (after BowmAN, 1969). 
In sub-auroral regions, spread- $F$ is recorded in association with both SAR arcs and SAI troughs. The oblique-angle ionospheric reflections which are associated with SAR arcs (KING and ROACH, 1961; WALKER and REES, 1968) suggest that height rises are involved. Also, as Fig. 16 shows, large height rises (with wavelike modulations of the equatorward edge) are associated with SAI troughs (BowMAN, 1969). Furthermore, both phenomena are accompanied by electron-density depletions, followed by (sometimes intense) spread-F occurrence (NAGY et al., 1970; RODGER and PINNOCK, 1982; RODGER, 1984). Thus the sequence of events for these two phenomena is very similar to the sequence of macroscale changes, discussed in other sections of this paper. If TIDs (generated by AGWs) are also associated with spread- $F$ occurrence in these latitude regions, as seems likely for mid-latitudes and equatorial latitudes, they may also in some way be associated with the macroscale changes, as has been suggested for the mid-latitude results. Speeds which range from $60 \mathrm{~ms}^{-1}$ to $126 \mathrm{~ms}^{-1}$ reported by BowMAN (1969) for the SAI trough are comparable with the speeds of medium-scale TIDs found elsewhere. Furthermore, it should be noted that KING and ROACH (1961) have suggested that SAR arcs are possibly due to ionospheric recombination, resulting from atmospheric mixing. Mixing due to the breaking of AGWs will be suggested in the following Discussion section as a mechanism for the generation of height rises and electron-density depletions.

\section{Discussion}

The $F$-region height oscillations (Table 1 ) and the wavy patterns recorded from satellite transmissions (OYA et al., 1982) are consistent with the passage of TID wavetrains. These events are recorded at times when spread- $F$ occurs, or can be expected. In view of experimental results and theoretical calculations (NEWTON et al., 1969; DYSON et al., 1970; CLARK and RAITT, 1976; HEDIN and MAYR, 1987) it seems reasonable to assume that these TIDs are produced by the passage of atmospheric gravity waves (AGWs) through the neutral atmosphere.

The existence of small-scale ionospheric stratifications in the middle atmosphere has been known for some time. Various methods of detection have been used, but most of the investigations have involved rocket flights (see e.g., WHALE, 1951; ELLYETT and WATTS, 1959; BEDINGER et al., 1968). Further, experimental evidence of small-scale stratifications was found recently in two middle-atmosphere regions. These were regions embracing the $D$-region and below (GOLDBERG et al., 1988; BLOOD et al., 1988) and the $E$-region (FRITTS et al., 1988). Vertical spacings of around $2 \mathrm{~km}$ were found for both regions. BowMAN (1989), using ionograms produced by the disonde, has detected $E$-layer stratifications on 132 occasions over a 14-day period with an average vertical spacing of approximately $2 \mathrm{~km}$. Figures $2(\mathrm{~h})$ and 2(i) illustrate this $E$-layer stratification. However, what is important to this present investigation is the fact that ionogram traces at higher levels of the ionosphere ( $E_{2}, F_{1}$ and $F_{2}$ layers) also show these stratification effects (BowMAN, 1987, 1988b, 1989; BowMAN et al., 1988). These stratifications have been classed as medium-scale ionospheric structures and are discussed in Subsection 2.2 (see Figs. 2(c), 2(d), 2(e) and 2(f)). These are detected on both daytime and nighttime ionograms. These effects are seen at times when TIDs are present and in particular when spread- $F$ is present (BOWMAN, 1987). Even in equatorial regions, rocket flights 
have detected quasi-periodic electron-density variations with height at $F_{2}$-region levels at times of spread- $F$ occurrence (VICKREY et al., 1984). Because of the substantial evidence for small-scale stratifications in middle-atmosphere regions, much theoretical work is proceeding to explain these results (see e.g., HINES, 1988); in particular, the breaking of AGWs at these levels has received much attention (FrITTS, 1984, 1985; SCHOEBERL, 1987; MURAOKA et al., 1988). The occurrence of harmonics of the vertical wavelength is predicted (WEINSTOCK, 1986, 1988).

In view of the stratifications which have been reported for the higher ionospheric levels (BOWMAN, 1987, 1988b, 1989; BOWMAN et al., 1988) it may be worthwhile to consider the possibility of breaking AGWs being responsible for some of the macroscale changes which are reported in this paper. NEWTON et al. (1969), who reported waves in the neutral atmosphere at $F$-region heights, states, "Apparently four integrally related wavelengths have been observed, a "fundamental" and the second, third and fourth harmonics," as might be expected if the gravity waves were breaking. Also, RoTTGER (1976) has suggested that harmonics generated by breaking AGWs may account for some of the equatorial spread- $F$ characteristics. Finally, KING $(1966,1971)$ has suggested that the $f_{\mathrm{o}} F_{2}$ depressions which occur during the passage of large-scale TIDs (as explained earlier) may be associated with the occurrence of negative ionospheric storms. Furthermore, he supports the idea that breaking AGWs are responsible for these depressions. Quoting from his 1966 paper, he states, "Waves travel through the ionospheric $F$-region producing several effects, the most tractable being the atmospheric mixing when the waves dissipate ... the wave energy is rapidly lost in turbulence ... mixing raises the recombination rate for ionospheric electrons so that $f_{0} F_{2}$ is depressed". BLANC and RICKEL (1989) have reported ionospheric irregularities at $F$-region levels which apparently are generated by nonlinear characteristics of acoustic waves propagated from ground level. In view of the foregoing, particularly the fact that higher harmonics are recorded for AGWs at $F$-region levels (NEWTON et al., 1969) it would seem appropriate that the theoretical work on AGW saturation effects for middle-atmosphere regions be extended to higher levels of the ionosphere. Also quantitative calculations on electron-density depletions, expected for breaking AGWs, would also appear desirable. In the past (see e.g., MARTYN, 1959) it has been generally assumed that the production of spread- $F$ was (by whatever mechanism) a direct result of layer rising. By contrast, in the ideas presented here it is suggested that the TIDs are responsible for the spread- $F$ traces, while at the same time producing the height rises because of electron-density depletions.

An AGW will break when its wave amplitude increases to a critical value (i.e., when wave saturation occurs). In the upper atmosphere, wave amplitudes will increase with height due to neutral-particle density decreases (HINES, 1960; MURAOKA et al., 1988) so that upward propagation may eventually lead to saturation. However, another mechanism which has been proposed for enhancing wave amplitudes is the spatial resonance mechanism (WHITEHEAD, 1971; BEER, 1973). KLOSTERMEYER (1978), ROTTGER (1978) and BOOKER (1979) have each proposed that this mechanism operates in equatorial regions to produce breaking AGWs (and associated TIDs) leading to some of the spread- $F$ structures observed in equatorial regions. In particular these authors suggest the initiation of the RayleighTaylor instability in the process leading to the "plumes" associated with the post- 
sunset spread-F. KERSLEY et al. (1980) have suggested that "medium scale gravity waves... breakdown to scintillation-producing irregularities by means of a spatial resonance" in an effort to explain the coexistence of waves in the TEC records and short-period scintillations (discussed earlier).

For spatial resonance to occur, it is necessary that the speeds and directions of travel of the gravity wave and the background wind be comparable. Klostermeyer, Rottger and Booker explain why it is likely that these conditions prevail in equatorial regions in the post-sunset period. If breaking AGWs are associated with the macroscale height rises and the accompanying electron-density depletions considered here for mid-latitude regions, it is worthwhile to consider whether or not the spatial resonance mechanism is likely to be operating in these regions. There is in fact some experimental evidence to suggest that this may be so. Table 2 of BowMAN (1968a) (see also BURKE, 1957) lists a number of events recorded at Brisbane where the speeds and directions of travel of (spread- $F$ associated) TIDs (as recorded by MCNICOL et al. (1956)) are matched by $F_{2}$-region drift movements in the same area. The movements in general were in directions in the north-west quadrant. In addition, sporadic- $E$-cloud movements and $E_{\mathrm{s}}$-layer drifts are consistent in speed and direction in the north-west quadrant (THOMAS and BURKE, 1956; BOWMAN, $1968 \mathrm{a})$. For another mid-latitude station near Melbourne, Australia $\left(37^{\circ} \mathrm{S}, 144^{\circ} \mathrm{E}\right.$ geographic, Dip $68^{\circ}-1500 \mathrm{~km}$ and approximately SSW from Brisbane, Dip 57 ) MORTON and ESSEX (1978) have also detected nighttime TIDs travelling in northwest directions with movements compatible with "prevailing background winds".

\section{Conclusions}

This paper reviews some recent publications on spread- $F$ in mid-latitude regions, and in addition presents some additional material to support these recent papers. The experimental evidence suggests that ionospheric structures associated with spread- $F$ occurrence might be divided into four different types of irregularities classified in terms of their sizes. These types are classed as "macroscale", "largescale", "medium-scale" and "small-scale". It is argued that the large-scale and medium-scale irregularities are the dominant structures in the creation of the extra traces known as mid-latitude spread- $F$ traces.

Some tens of minutes before the commencement of the mid-latitude spread- $F$ events discussed here, what appear to be TID wavetrains are observed. Probably as a result of the passage of these TIDs, there is a significant height rise (sometimes preceded by a small height suppression) which is modulated with wavetrain periodicities of around 20 minutes. Following this height rise, the layer tends to return to its original height, with oscillatory patterns sometimes also occurring during this phase of the height movements. The maximum electron density of the layer is reduced during the height rise with a recovery towards its original value in the following downward movement phase. It seems likely that the spread- $F$ which is usually seen during the recovery phase results from off-vertical reflections from the wavelike structures detected during the height rise, after these structures have moved a sufficient distance from the overhead positions to produce traces on ionograms with sufficient extra range (compared with the main trace) that spread- $F$ is observed on ionograms (see BoWMAN and MONRO, 1988). The additional slope produced by 
the macroscale height rise thus enhances the tilts of the oscillatory structures, so that spread- $F$ is more likely to be seen as the structures move away (diverging traces) rather than as they advance (MCNICOL et al., 1956). Figure 17 is a model representing the features of a spread- $F$ event, as suggested by the results presented here. There is a possibility that the mechanism responsible for the sequence of events reported here associated with mid-latitude spread- $F$ occurrence may apply more generally to phenomena at other latitude regions (Figs. 15 and 16 should be compared with Fig. 17).

The $F_{2}$-region stratifications (related to the medium-scale irregularities) combined with the observation of TID wavetrains associated with electron-density depletions (and related height rises) are all consistent with breaking AGWs being involved in leading to corresponding effects on the TIDs generated by the AGWs.

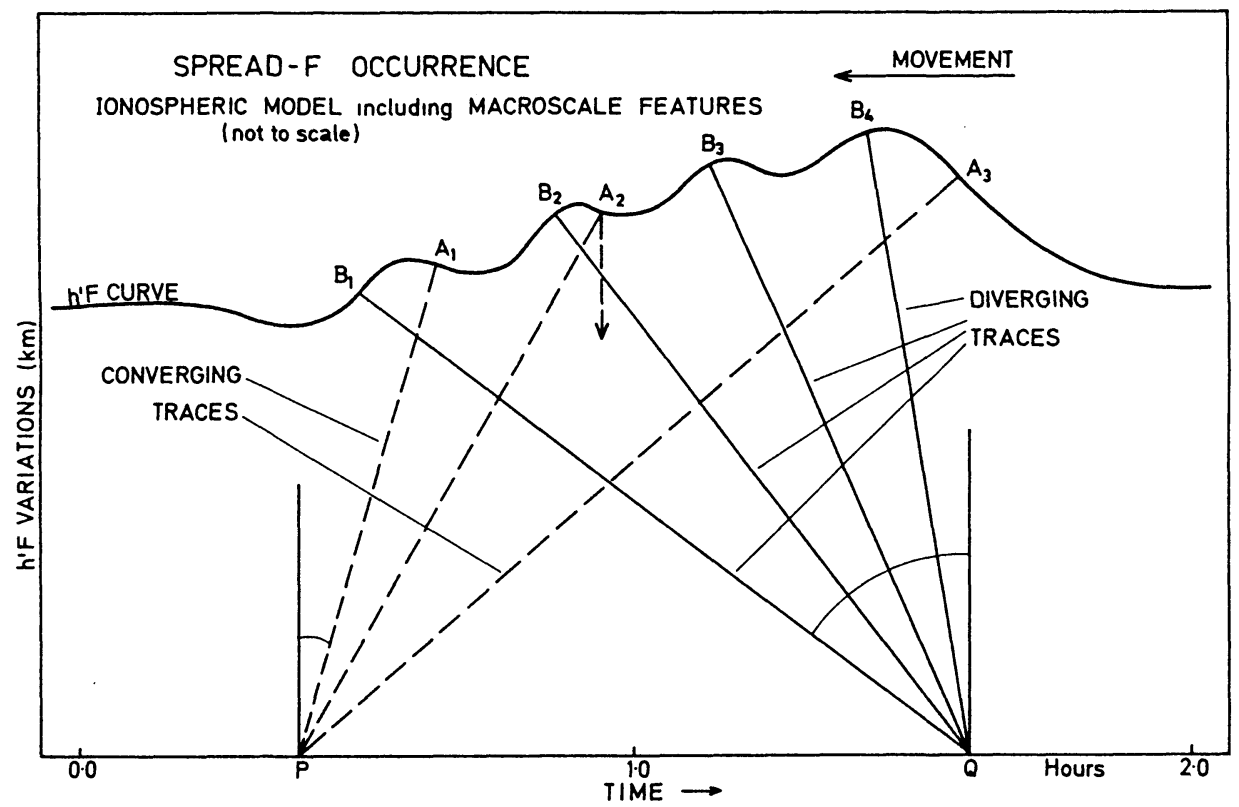

Fig. 17. Model of macroscale changes associated with mid-latitude spread- $F$ events. Also illustrated, proposed converging trace reflections at time $\mathbf{P}$ and proposed diverging trace reflections at time $\mathbf{Q}$.

\section{REFERENCES}

Alex, S., P. V. Koparkar, and R. G. Rastogi, Spread-F and ionization anomaly belt, J. Atmos. Terr. Phys., 51, 371-379, 1989.

ARgo, P. E. and M. C. Kelley, Digital ionosonde observations during equatorial spread-F, J. Geophys. Res., 91, 5539-5555, 1986.

BATES, H. F., The height of $F$-layer irregularities in the arctic ionosphere, J. Geophys. Res., 64, $1257-$ $1265,1959$.

Bates, H. F., The aspect sensitivity of spread-F irregularities, J. Atmos. Terr. Phys., 33, 111-115, 1971.

BATES, H. F. and P. R. AlbEe, Aspect sensitivity of F-layer HF backscatter echoes, J. Geophys. Res., 75, 165-170, 1970. 
Bedinger, J. F., H. Knaflich, E. Manring, and D. Layzer, Upper-atmosphere winds and their interpretation-I, Planet. Space Sci., 16, 159-193, 1968.

BEER, T., Spatial resonance in the ionosphere, Planet. Space Sci., 21, 297-307, 1973.

Berkner, L. V. and H. W. Wells, F-region ionospheric investigations at low latitudes, Terr. Magn. Atmos. Electr., 39, 215-230, 1934.

Blanc, E. and D. Rickel, Nonlinear wave fronts and ionospheric irregularities observed by HF sounding over a powerful acoustic source, Radio Sci., 24, 279-288, 1989.

Blood, S. P., J. W. Mitchell, C. L. Croskey, T. D. Raymund, E. V. Thrane, T.A. Blix, U. P. Hoppe, D. C. FRITTS, and F. J. SchmidLin, Studies of high latitude mesospheric turbulence by radar and rocket 2: Measurements of small scale turbulence, J. Atmos. Terr. Phys., 50, 963-976, 1988.

BOOKER, H. G., The role of acoustic gravity waves in the generation of spread- $F$ and ionospheric scintillation, J. Atmos. Terr. Phys., 41, 501-515, 1979.

Booker, H. G. and H. W. Wells, Scattering of radio waves by the $F$-region of the ionosphere, Terr. Magn. Atmos. Electr., 43, 249-256, 1938.

Booker, H. G., P. K. Pasricha, and W. J. Powers, Use of scintillation theory to explain frequencyspread on F-region ionograms, J. Atmos. Terr. Phys., 48, 327-354, 1986.

Bowman, G. G., Further studies of "spread-F" at Brisbane, I-Experimental, Planet. Space Sci., 2 , 150-156, 1960a.

Bowman, G. G., A relationship between "spread- $F$ " and the height of the $F_{2}$ ionospheric layer, Aust. J. Phys., 13, 69-72, 1960b.

Bowman, G. G., Some effects of nuclear explosions on the ionosphere, Aust. J. Phys., 15, 405-419, 1962.

Bowman, G. G., Spread- $F$ in the ionosphere and the neutral particle density of the upper atmosphere, Nature, 201, 564-566, 1964.

Bowman, G. G., Travelling disturbances associated with ionospheric storms, J. Atmos. Terr. Phys., 27, 1247-1261, 1965.

Bowman, G. G., Movements of ionospheric irregularities and gravity waves, J. Atmos. Terr. Phys., 30, 721-734, 1968a.

Bowman, G. G., The nature of spread-F irregularities in Antarctica, Aust. J. Phys., 21, 695-714, 1968 b.

Bowman, G. G., Ionization troughs below the $F_{2}$-layer maximum, Planet. Space Sci., 17, 777-796, 1969.

Bowman, G. G., Low-latitude ionospheric height changes associated with geomagnetic storms, J. Atmos. Terr. Phys., 39, 1169-1173, 1977.

Bowman, G. G., A relationship between polar magnetic substorms, ionospheric height rises and the occurrence of spread-F, J. Atmos. Terr. Phys., 40, 999-1004, 1978.

Bowman, G. G., The nature of ionospheric spread- $F$ irregularities in mid-latitude regions, J. Atmos. Terr. Phys., 43, 65-79, 1981.

Bowman, G. G., A comparison of mid-latitude and equatorial-latitude spread- $F$ characteristics, $J$. Atmos. Terr. Phys., 46, 65-71, 1984.

Bowman, G. G., Some $F_{2}$ layer-sporadic-E relationships, Ann. Geophys., 4A, 55-60, 1986.

Bowman, G. G., Small scale stratification of the mid-latitude F-region, J. Atmos. Terr. Phys., 49, 1007-1015, 1987.

Bowman, G. G., Large-scale ionospheric structures associated with mid-latitude spread-F, J. Geophys. Res., 93, 5955-5958, 1988a.

Bowman, G. G., Small-scale stratifications associated with daytime travelling ionospheric disturbances in mid-latitude regions, Ann. Geophys., 6, 187-194, 1988b.

Bowman, G. G., Some aspects of small-scale stratifications at $E$-layer and $F_{2}$-layer levels of the daytime mid-latitude ionosphere, Ann. Geophys., 7, 77-82, 1989.

Bowman, G. G. and D. W. HaInswORTH, Weak daytime spread- $F$ signals from the mid-latitude ionosphere, Ann. Geophys., 5A, 127-132, 1987.

Bowman, G. G., D. W. Hainsworth, and G. S. Dunne, A research-oriented ionosonde with directional capabilities, Radio Sci., 21, 297-303, 1986.

Bowman, G. G. and P. E. Monro, Mid-latitude range spread and travelling ionospheric disturbances, $J$. Atmos. Terr. Phys., 50, 215-223, 1988.

Bowman, G. G. and K. L. Shrestha, Ionospheric storms and small pressure fluctuations at ground level, Nature, 210, 1032-1034, 1966.

Bowman, G. G., G. S. Dunne, and D. W. Hainsworth, Mid-latitude spread-F occurrence during daylight hours, J. Atmos. Terr. Phys., 49, 165-176, 1987. 
Bowman, G. G., R. H. Clarke, and D. H. Meehan, Mid-latitude frequency spread and its association with small-scale ionospheric stratifications, J. Atmos. Terr. Phys., 50, 797-809, 1988.

BriggS, B. H., Observations of radio star scintillations and spread- $F$ echoes over a solar cycle, J. Atmos. Terr. Phys., 26, 1-23, 1964.

BURKE, M. J., Measurement of horizontal motion in the ionosphere, M. Sc. Thesis, The University of Queensland, Brisbane, Australia, 1957.

Clark, D. H. and W. J. RaITT, Wave-like structure in the ionosphere at low and middle latitudes, $J$. Atmos. Terr. Phys., 38, 1245-1250, 1976.

ClarKe, R. H., Ground-based radio observations of middle-latitude ionospheric irregularities, Ph.D. Thesis, The University of Queensland, Brisbane, Australia, 1972.

Dyson, P. L., G. P. Newton, and L. H. Brace, In situ measurements of neutral and electron density wave structure from the Explorer 32 satellite, J. Geophys. Res., 75, 3200-3210, 1970.

Ellyett, C. and J. M. WATTS, Stratification in the lower ionosphere, J. Res. NBS, 63D, 117-134, 1959.

FeJer, B. G. and M. C. Kelley, Ionospheric irregularities, Rev. Geophys. Space Phys., 18, 401-454, 1980.

Francis, S. H., Global propagation of atmospheric gravity waves: A review, J. Atmos. Terr. Phys., 37, 1011-1054, 1975.

FRITTS, D. C., Gravity wave saturation in the middle atmosphere: A review of theory and observations, Rev. Geophys. Space Phys., 22, 275-308, 1984.

FritTS, D. C., A numerical study of gravity wave saturation: Nonlinear and multiple wave effects, $J$. Atmos. Sci., 42, 2043-2058, 1985.

Fritts, D. C., S. A. Smith, B. B. Balsley, and C. R. Philbrick, Evidence of gravity wave saturation and local turbulence production in the summer mesosphere and lower thermosphere during the STATE experiment, J. Geophys. Res., 93, 7015-7025, 1988.

From, W. R. and D. H. Meehan, Mid-latitude spread-F structure, J. Atmos. Terr. Phys., 50, 629-638, 1988.

Goldberg, R. A., D. C. Fritts, H. G. Chou, J. R. Barcus, and F. J. Schmidlin, Studies of high latitude mesopheric turbulence by radar and rocket 1: Energy deposition and wave structure, $J$. Atmos. Terr. Phys., 50, 951-961, 1988.

HaJkowiCZ, L. A., Conjugate effects in the generation of travelling ionospheric disturbances (TIDs) in the F-region, Planet. Space Sci., 31, 1409-1413, 1983.

Hajkowicz, L. A. and R. D. Hunsucker, A simultaneous observation of large-scale periodic TIDs in both hemispheres following an onset of auroral disturbances, Planet. Space Sci., 35, 785-791, 1987.

HAKURA, Y., Tables and maps of geomagnetic coordinates corrected by the higher order spherical harmonic terms, J. Radio Res. Lab. (Japan), 12, 121-155, 1965.

Hedin, A. E. and H. G. MAYR, Characteristics of wavelike fluctuations in dynamics explorer neutral composition data, J. Geophys. Res., 92, 11159-11172, 1987.

Herman, J. R., Spread-F and ionospheric F-region irregularities, Rev. Geophys., 4, 255-299, 1966.

Hines, C. O., Internal gravity waves at ionospheric heights, Can. J. Phys., 38, 1441-1481, 1960.

Hines, C. O., Generation of turbulence by atmospheric gravity waves, J. Atmos. Sci., 45, 1269-1278, 1988.

KAUShIKA, N. D. and F. DE MENDONCA, Nighttime fluctuations (scintillations) in Faraday rotation angle of VHF signals from geostationary satellites, Planet. Space Sci., 22, 1331-1334, 1974.

Kelley, M. C., M. F. Larsen, C. LaHoz, and J. P. McClure, Gravity wave initiation of equatorial spread-F: A case study, J. Geophys. Res., 86, 9087-9100, 1981.

Kersley, L., J. Aarons, and J. A. Klobuchar, Nighttime enhancements in total electron content near Arecibo and their association with VHF scintillations, J. Geophys. Res., 85, 4214-4222, 1980.

KING, G. A. M., The ionospheric disturbance and atmospheric waves, I-general discussion, J. Atmos. Terr. Phys., 28, 957-963, 1966.

KIng, G. A. M., Spread-F on ionograms, J. Atmos. Terr. Phys., 32, 209-221, 1970.

KIng, G. A. M., The ionospheric F-region storm, J. Atmos. Terr. Phys., 33, 1223-1240, 1971.

KING, G. A. M. and F. E. ROACH, Relationship between red auroral arcs and ionospheric recombination, J. Res. NBS, 65D, 129-135, 1961

KLemperer, W. K., Characteristics of spread- $F$ at high geomagnetic latitude, J. Geophys. Res., 68, 3191-3196, 1963.

KLOSTERMEYER, J., Nonlinear investigation of the spatial resonance effect in the nighttime equatorial 
F-region, J. Geophys. Res., 83, 3753-3760, 1978.

LeI, Y. H., X. Q. PI, D. Y. YE, and B. X. LIANG, Nocturnal ionospheric scintillation, spread-F and TEC wave-like perturbation at Wuchang, Proc. Int. Symp. Space Phys., Beijing, November, 1986, pp. 5-054-5-057, 1986.

LobB, R. J. and J. E. Titheridge, The effects of travelling ionospheric disturbances on ionograms, $J$. Atmos. Terr. Phys., 39, 129-138, 1977.

MARTYN, D. F., Large-scale movements of ionization in the ionosphere, J. Geophys. Res., 64, 2178-2179, 1959.

MCNicol, R. W. E., H. C. Webster, and G. G. Bowman, A study of "spread- $F$ " ionospheric echoes at night at Brisbane, I, Range spreading (experimental), Aust. J. Phys., 9, 247-271, 1956.

Morton, F. W. and E. A. Essex, Gravity wave observations at a southern hemisphere mid-latitude station using the total electron content technique, J. Atmos. Terr. Phys., 40, 1113-1122, 1978.

Munro, G. H., Reflections from irregularities in the ionosphere, Proc. Roy. Soc., A219, 447-463, 1953.

Muraoka, Y., T. Sugiyama, K. Kawahira, T. Sato, T. Tsuda, S. Fukao, and S. Kato, Cause of a monochromatic inertia-gravity wave breaking observed by the MU radar, Geophys. Res. Lett., 15, 1349-1352, 1988.

Nagy, A. F., R. G. Roble, and P. B. Hays, Stable mid-latitude red arcs; observations and theory, Space Sci. Rev., 11, 709-727, 1970.

Newton, G. P., D. T. Pelz, and H. Volland, Direct in situ measurements of wave propagation in the neutral thermosphere, J. Geophys. Res., 74, 183-196, 1969.

Oya, H., T. TAKahashi, A. Morioka, and H. MiyaOKa, Wavy patterns of ionospheric electron density profiles triggered by TID-Observation results of the electron density by Taiyo satellite, J. Geomag. Geoelectr., 34, 509-526, 1982.

RASTOGI, R. G., Early phase of equatorial spread- $F$ seen by HF ionosonde and VHF backscatter radar, Indian J. Radio Space Phys., 10, 148-152, 1981.

Rastogi, R. G., Features of spread-F at Grand Bahama, a temperate latitude station, Ann. Geophys., 7 , 177-182, 1989.

Rastogi, R. G., P. V. Koparkar, H. Chandra, and G. D. Vyas, Spread- $F$ and radio wave scintillations near the $F$-region anomaly crest, Ann. Geophys., 7, 281-284, 1989.

Rodger, A. S., Spread- $F$ ionospheric irregularities and their relationship to stable auroral red arcs at magnetic mid-latitudes, J. Atmos. Terr. Phys., 46, 335-342, 1984.

Rodger, A. S. and J. Aarons, Studies of ionospheric $F$-region irregularities from geomagnetic midlatitude conjugate regions, J. Atmos. Terr. Phys., 50, 63-72, 1988.

Rodger, A. S. and M. Pinnock, Movements of the mid-latitude ionospheric trough, J. Atmos. Terr. Phys., 44, 985-992, 1982.

RotTger, J., Wavelike structures of large scale equatorial spread-Firregularities, J. Atmos. Terr. Phys., 35, 1195-1206, 1973.

RotTger, J., The macro-scale structure of equatorial spread-F irregularities, J. Atmos. Terr. Phys., 38, 97-101, 1976.

RoTTGER, J., Drifting patches of equatorial spread- $F$ irregularities-experimental support for the spatial resonance mechanism in the ionosphere, J. Atmos. Terr. Phys., 40, 1103-1112, 1978.

Roy, R. and J. K. W. Verma, Irregularities in the ionosphere, J. Geophys. Res., 58, 473-485, 1953.

Schmerling, E. R., Ten-point method of ionogram reduction, Radio Sci., 2, 1233-1236, 1967.

Schoeberl, M. R., Dynamics of the middle atmosphere, Rev. Geophys., 25, 501-507, 1987.

ShimAZAKI, T., A statistical study of occurrence probability of spread-F at high latitudes, J. Geophys. Res., 67, 4617-4634, 1962.

SINNO, K. and M. KAN, Mid-latitude ionospheric scintillations of VHF radio signals associated with peculiar fluctuations of Faraday rotation, J. Atmos. Terr. Phys., 40, 503-506, 1978.

SkinNer, N. J. and R. F. Kelleher, Studies of $F$-region irregularities at Nairobi 1 -from spread- $F$ on ionograms 1964-1970, Ann. Geophys., 27, 181-194, 1971.

Sterling, W. L., W. H. Hooke, and R. Cohen, Traveling ionospheric disturbances observed at the magnetic equator, J. Geophys. Res., 76, 3777-3782, 1971.

TAKahashi, T., H. OYa, and S. WaTAnabe, Ionospheric disturbances induced by substorm associated electric fields in the low-latitude F-region, J. Geomag. Geoelectr., 39, 187-209, 1987.

TANAKA, T., An important role of electric field reversals for the initiation of gigahertz scintillations at midlatitude during geomagnetic storms, J. Geomag. Geoelectr., 39, 659-676, 1987. 
Thomas, J. A. and M. J. Burke, Motion in the nighttime $E_{\mathrm{s}}$ region at Brisbane, Aust. J. Phys., 9, 440-453, 1956.

Tsunoda, R. T., Time evolution and dynamics of equatorial backscatter plumes, 1. Growth phase, $J$. Geophys. Res., 86, 139-149, 1981.

TSUNODA, R. T., On the generation and growth of equatorial backscatter plumes, 2. Structuring of the west wall of upwellings, J. Geophys. Res., 88, 4869-4874, 1983.

Tsunoda, R. T., R. C. Livingston, J. P. McClure, and W. B. Hanson, Equatorial plasma bubbles; vertically elongated wedges from the bottomside $F$ layer, J. Geophys. Res., 87, 9171-9180, 1982.

UyedA, H. and Y. Ogata, An example of the records of the travelling $F_{2}$ layer in the nighttime, Rep. Ionos. Res. Jpn., 8, 103-107, 1954.

Vickrey, J. F., M. C. Kelley, R. Pfaff, and S. R. Goldman, Low-latitude image striations associated with bottomside equatorial spread-F; observations and theory, J. Geophys. Res., 89, 2955-2961, 1984.

Walker, J. C. G. and M. H. ReES, Excitation of stable auroral red arcs at sub-auroral latitudes, Planet. Space Sci., 16, 915-928, 1968.

Weber, E. J., J. Buchau, R. H. Eather, and S. B. Mende, North-south aligned equatorial airglow depletions, J. Geophys. Res., 83, 712-716, 1978,

Weinstock, J., Finite amplitude gravity waves: Harmonics, advective steepening and saturation, $J$. Atmos. Sci., 43, 688-704, 1986.

Weinstock, J., Saturation of gravity waves, in Handbook for Middle Atmosphere Program, Vol. 26, edited by B. Edwards, p. 66, Scientific Committee on Solar-Terrestrial Physics (SCOSTEP), Urbana, Illinois, 1988.

Whale, H. A., Fine structure of the ionospheric region E, J. Atmos. Terr. Phys., 1, 233-243, 1951.

WhITEHEAD, J. D., Ionization disturbances caused by gravity waves in the presence of an electrostatic field and background wind, J. Geophys. Res., 76, 238-241, 1971.

WhiteheAD, J. D. and E. KANTARIzis, Errors in the measurement of virtual height using a phase ionosonde, J. Atmos. Terr. Phys., 29, 1483-1488, 1967. 